\begin{tabular}{|c|c|}
\hline Title & The novel heart-specific RING finger protein 207 is involved in energy metabolism in cardiomy ocytes \\
\hline Author(s) & $\begin{array}{l}\text { Mizushima, W ataru; T akahashi, Hidehisa; W atanabe, Masashi; Kinugawa, Shintaro; Matsushima, Shouji; Takada, } \\
\text { Shingo; Y okota, T akashi; Furihata, T akaaki; Matsumoto, Junichi; T suda, Masay a; Chiba, Ikuru; Nagashima, Shun; } \\
\text { Y anagi, Shigeru; Matsumoto, Masaki; Nakay ama, Keiichi I.; T sutsui, Hiroyuki; Hatakeyama, Shigetsugu }\end{array}$ \\
\hline Citation & $\begin{array}{l}\text { Journal of molecular and cellular cardiology, 100, } 43-53 \\
\text { https://doi.org/10.1016/ .yjmcc.2016.09.013 }\end{array}$ \\
\hline Issue Date & 2016-11 \\
\hline Doc URL & http:/hdl.handle.net/2115/67491 \\
\hline Rights & $\begin{array}{l}\text { (c) 2016. This manuscript version is made available under the CC-BY-NC-ND } 4.0 \text { license } \\
\text { http://creativecommons.org/icenses/by-nc-nd/4.0/ }\end{array}$ \\
\hline Rights(URL) & http://creativecommons.org/icenses/by-nc-nd/4.0/ \\
\hline Type & article (author version) \\
\hline File Information & R207_JMCC.pdf \\
\hline
\end{tabular}

Instructions for use 


\title{
The novel heart-specific RING finger protein 207 is involved in energy metabolism in cardiomyocytes
}

\author{
Wataru Mizushima ${ }^{\text {a,b }}$, Hidehisa Takahashi ${ }^{\text {b, Masashi Watanabe }}{ }^{\text {b }}$, Shintaro \\ Kinugawa a, Shouji Matsushima ${ }^{\text {a }}$, Shingo Takada a, Takashi Yokota ${ }^{\text {a }}$, Takaaki \\ Furihata $^{\text {a }}$, Junichi Matsumoto ${ }^{\text {a }}$, Masaya Tsuda ${ }^{\text {a }}$, Ikuru Chiba ${ }^{\text {b }}$, Shun Nagashima \\ ${ }^{\text {c, Shigeru Yanagi }}{ }^{\mathrm{c}}$, Masaki Matsumoto ${ }^{\mathrm{d}}$, Keiichi I. Nakayama ${ }^{\mathrm{d}}$, Hiroyuki Tsutsui \\ a , Shigetsugu Hatakeyama ${ }^{\text {b,* }}$
}

${ }^{a}$ Department of Cardiovascular Medicine, Hokkaido University Graduate School of Medicine, Kita 15, Nishi 7, Kita-ku, Sapporo, Hokkaido 060-8638, Japan

${ }^{b}$ Department of Biochemistry, Hokkaido University Graduate School of Medicine, Kita 15, Nishi 7, Kita-ku, Sapporo, Hokkaido 060-8638, Japan

${ }^{c}$ Laboratory of Molecular Biochemistry, School of Life Sciences, Tokyo University of Pharmacy and Life Sciences, Hachioji, Tokyo 192-0392, Japan

${ }^{d}$ Department of Molecular and Cellular Biology, Medical Institute of Bioregulation, Kyushu University, Maidashi 3-1-1, Higashi-ku, Fukuoka, Fukuoka 812-8582, Japan

*Corresponding author. Dr. Shigetsugu Hatakeyama, Department of Biochemistry, Hokkaido University Graduate School of Medicine, Kita 15 Nishi 7, Kita-ku, Sapporo, Hokkaido 060-8638, Japan

Tel: +81-11-706-5899; Fax: +81-11-706-5169

E-mail: hatas@med.hokudai.ac.jp

Keywords: cardiomyocyte, RNF207, energy metabolism, VDAC, heart failure 


\begin{abstract}
A failing heart shows severe energy insufficiency, and it is presumed that this energy shortage plays a critical role in the development of cardiac dysfunction. However, little is known about the mechanisms that cause energy metabolic alterations in the failing heart. Here, we show that the novel RING-finger protein 207 (RNF207), which is specifically expressed in the heart, plays a role in cardiac energy metabolism. Depletion of RNF207 in neonatal rat cardiomyocytes (NRCs) leads to a reduced cellular concentration of adenosine triphosphate (ATP) and mitochondrial dysfunction. Consistent with this result, we observed here that the expression of RNF207 was significantly reduced in mice with common cardiac diseases including heart failure. Intriguingly, proteomic approaches revealed that RNF207 interacts with the voltage-dependent anion channel (VDAC), which is considered to be a key regulator of mitochondria function, as an RNF207-interacting protein. Our findings indicate that RNF207 is involved in ATP production by cardiomyocytes, suggesting that RNF207 plays an important role in the development of heart failure.
\end{abstract}




\section{Highlights}

- RNF207 is specifically expressed in the heart.

- RNF207 enhances activity of the tricarboxylic acid cycle in cardiomyocytes.

- RNF207 directly interacts with VDAC1 through its coiled-coil domain.

- Heart failure significantly reduces the expression of RNF207 in mice. 


\section{Introduction}

The number of people suffering from heart failure (HF) has been increasing worldwide, especially in countries with markedly aging populations [1]. Although pharmacological and device-based therapies for HF have made dramatic progress over the past few decades, the one-year mortality rate for HF is still high at $10 \%$ [2]. The heart beats approximately 100,000 times per day, and the oxygen consumption of the heart accounts for $10 \%$ of the body's total oxygen consumption [3]. To meet this tremendous energy demand, the body's cardiac metabolism is strictly regulated under normal conditions [4-6].

The cardiac metabolism in a failing heart is different from that in a normal heart. The metabolic changes observed in HF are defined by reduced cardiac energy production that may arise from progressive disorder in substrate utilization and mitochondrial function $[3,7]$. Since the heart demands large amounts of energy to maintain contractile function, a reduced production of adenosine triphosphate (ATP) may directly lead to contractile dysfunction [8]. It is thus rational to assume that the cardiac metabolism plays an important role in the pathophysiology of HF.

Two of the most common cardiac pathologies that eventually lead to HF are (1) cardiac hypertrophy induced by pressure overload and (2) ischemia/reperfusion injury, and it has also been reported that cardiac metabolic changes occur in a condition called the 'compensated state' [7,9]. Although the clarification of metabolic changes in HF has progressed, little is known about the mechanisms that cause energy metabolic alterations in HF. Elucidation of the mechanisms through which cardiac metabolic changes occur in HF could thus uncover new therapeutic strategies for HF. 
The really interesting new gene (RING)-finger domain contains eight metal-binding residues that coordinate two zinc atoms for protein folding and biological function [10]. More than 200 proteins containing a RING-finger domain (RNF) are encoded in the human genome. It has been reported that several RNF proteins play essential roles in many cellular processes including signal transduction [11], apoptosis [12] and lipid metabolism [13]. It was also recently demonstrated that mutations of some RNF proteins are involved in colorectal cancer [14], RIDDLE syndrome [15] and Moyamoya disease [16]. The RNF proteins RNF41 and RNF146 were reported to be associated with the development of cardiac dysfunction $[17,18]$. It has been shown that some of the RNF proteins are tissue-specifically expressed [19] and that the mRNA of RNF207 is specifically expressed in the heart $[20,21]$. However, the detailed function of RNF207 in the heart has not been elucidated.

The VDAC was first characterized as a mitochondrial outer membrane protein and it has three isoforms in humans: VDAC1, VDAC2 and VDAC3 [22]. The VDAC was initially considered to be a channel through which ions and metabolites such as ATP, adenosine diphosphate (ADP) and nicotinamide adenine dinucleotide (NADH) pass between the cytosol and mitochondria, but recent studies have shown that VDACs play an essential role in energy homeostasis, selective mitochondrial autophagy (mitophagy) and calcium homeostasis [23-25].

In the present study, we found that RNF207 protein is specifically expressed in the heart. A depletion of RNF207 in neonatal rat cardiomyocytes (NRCs) induced a reduction of ATP concentration and mitochondrial dysfunction. RNF207 interacts with the outer mitochondrial membrane protein VDAC1. The expression of RNF207 was significantly reduced in mouse models of common heart diseases including cardiac 
hypertrophy, ischemic-reperfusion injury and HF. Taken together, these findings suggest that RNF207 is involved in cardiac energy metabolism and the pathogenesis of common heart diseases. 


\section{Materials and Methods}

\subsection{Cloning and plasmid construction}

We amplified mouse Rnf207 cDNAs from a mouse heart cDNA library by polymerase chain reaction (PCR) with BlendTaq (Takara, Tokyo) using the following primers: 5'-TAAATGTCTGGAGCAATTTTTGCG-3' (RNF207 forward), 5'-TTGTCAGGCTTGGTAGCAAGCCCC-3' (RNF207 reverse). We amplified mouse Vdacl cDNAs from cDNAs of mouse myoblast $\mathrm{C} 2 \mathrm{C} 12$ cells by PCR with BlendTaq (Takara) using the following primers: 5'-ATGGCCGTGCCTCCCACATA-3' (VDAC1 forward) and 5'-TTATGCTTGAAATTCCAGTCCTAGG-3' (VDAC1 reverse). We subcloned the amplified fragments into pBluescript II SK(+) (Stratagene, La Jolla, CA) and verified the sequences. The resulting cDNA fragments were subcloned into the vectors pCGN-HA, p3×FLAG, p3×FLAG-CMV7.1, pcDNA3.1/myc-His and pMX-puro. Rnf207 cDNAs including deletion mutants and point mutations and Vdacl cDNAs including deletion mutants were amplified by PCR and subcloned into p3 $\times$ FLAG and p3×FLAG-CMV7.1.

\subsection{Cell culture}

Human embryonic kidney 293T (HEK293T) cells, mouse myoblast C2C12 cells (ATCC, Manassas, VA) and their derivatives were cultured under an atmosphere of $5 \%$ $\mathrm{CO}_{2}$ at $37^{\circ} \mathrm{C}$ in Dulbecco's modified Eagle's medium (DMEM) (Sigma-Aldrich, St. Louis, MO) supplemented with 10\% (v/v) fetal bovine serum (Invitrogen, Carlsbad, 
CA), $55 \mu \mathrm{M} \beta$-mercaptoethanol (GIBCO, Grand Island, NY), 2 mM L-glutamine, 0.1 $\mathrm{mM}$ MEM non-essential amino acid, penicillin $\left(10 \mathrm{U} \mathrm{ml}^{-1}\right)$ and streptomycin $(0.1 \mathrm{mg}$ $\mathrm{ml}^{-1}$ ) (Sigma). Cells stably overexpressing $3 \times$ Flag-RNF207 were established by a

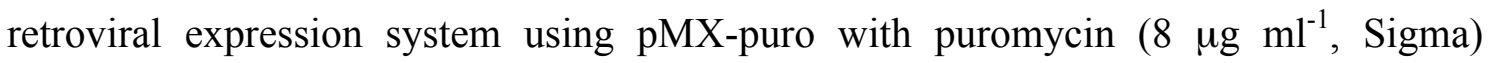
selection as described previously [26].

\subsection{Primary culture of rat neonatal ventricular myocytes}

We prepared ventricular cardiomyocytes from 2-3-day-old Crl:(WI) BR-Wistar rats (Sankyou Labo Service, Tokyo). A cardiomyocyte-rich fraction was obtained by centrifugation through a discontinuous Percoll gradient as described previously [27].

\subsection{Mice}

C57BL/6J mice were purchased from CLEA Japan (Tokyo). The mice were used for experiments at 10-12 weeks of age (weight, 23-27 g). The mice were bred in a pathogen-free environment and kept under a constant 12-h light-dark cycle at a temperature of $23-25^{\circ} \mathrm{C}$. Standard chow and water were provided. All of the animal protocols were reviewed and approved by the Animal Welfare Committee of Hokkaido University. The work presented in this study is covered by the Animal Protocol No.APN-13-0074. All of the research staff who performed the procedures using live animals were pre-approved by the Animal Welfare Committee of Hokkaido University based on their completion of required animal use and care training and experience in animal experiments. 


\subsection{Mass spectrometry analysis}

Immunoprecipitated proteins were separated by sodium dodecyl sulfate-polyacrylamide gel electrophoresis (SDS-PAGE) and stained with silver staining. The silver-stained bands were excised from the gel. The proteins therein were subjected to in-gel reduction, S-carboxyamidomethylation and digestion with sequence-grade trypsin (Promega, Fitchburg, WI) as described previously [28]. The resultant peptides were analyzed by liquid chromatography-electrospray ionization-tandem mass spectrometry (LC ESI-MS/MS) (LCQ DECA and LTQ XL; Thermo Fisher Scientific, Waltham, MA). The data were analyzed using Mascot software (Matrix Science, Boston, MA).

\subsection{Transfection, immunoprecipitation and Western blot analysis}

Cells were transfected by Fugene HD reagent (Roche, Branchburg, NJ) and lysed in a solution containing $50 \mathrm{mM}$ Tris- $\mathrm{HCl}(\mathrm{pH} 7.6), 150 \mathrm{mM} \mathrm{NaCl}, 0.5 \%$ Triton $\mathrm{X}-100$, leupeptin $\left(10 \mu \mathrm{g} \mathrm{ml}^{-1}\right), 1 \mathrm{mM}$ phenylmethylsulfonyl fluoride, $400 \mu \mathrm{M} \mathrm{Na}_{3} \mathrm{VO}_{4}, 400 \mu \mathrm{M}$ EDTA, $10 \mathrm{mM} \mathrm{NaF}$, and $10 \mathrm{mM}$ sodium pyrophosphate. The cell lysates were centrifuged at $16,000 \mathrm{~g}$ for $15 \mathrm{~min}$ at $4^{\circ} \mathrm{C}$, and the resulting supernatant was collected. Protein concentrations were measured (Pierce BCA, Rockford, IL, USA) and lysates that had the same amounts of protein were incubated with anti-FLAG M2 agarose (Sigma) or anti-HA-agarose (Sigma) for $1 \mathrm{~h}$ at $4^{\circ} \mathrm{C}$.

The beads was separated by centrifugation and washed five times with ice-cold 
lysis buffer, and the protein complexes were then eluted by $3 \times$ FLAG peptide (Sigma) or $3 \times$ HA peptide (Sigma). Immune complexes were detected with primary antibodies, horseradish peroxidase-conjugated antibodies to mouse or rabbit IgG (GE Healthcare Bioscience, Princeton, NJ) and an enhanced chemiluminescence system (GE Healthcare).

\subsection{Antibody generation}

Glutathione $S$-transferase (GST)-fusion proteins including 117-141 amino acid residues of mouse RNF207 were expressed in XL-10 cells using the pGEX4T-2 plasmid vector (GE Healthcare) and then purified by reduced glutathione-Sepharose beads (GE Healthcare). The recombinant protein was used for immunization in rabbits. A rabbit polyclonal anti-RNF207 antibody was affinity-purified using a recombinant RNF207-conjugated Sepharose 4B column.

\subsection{Recombinant protein and in vitro binding assay}

GST-fused protein of mouse VDAC1 was expressed in XL-10 cells using the pGEX6P-1 plasmid vector (GE Healthcare) and then purified by reduced glutathione-sepharose beads (GE Healthcare). His 6 -Flag-tagged mouse RNF207 proteins including deletion mutants were expressed in Rosetta blue cells with the use of the pET30 plasmid vector (Novagen, Madison, WI) and then purified with the use of ProBond metal affinity beads (Invitrogen).

For the production of recombinant proteins in Sf9 cells, we subcloned full-length 
mouse Rnf207 cDNAs into pFastBac HTc with epitope tags and expressed epitope-tagged full-length mouse Rnf207 with the BAC-to-BAC system (Clontech Laboratories, Mountain View, CA). Baculovirus infections, culture and affinity purifications were performed as described previously [29]. In vitro binding assays were performed as described previously [30]

\subsection{Antibodies}

We used anti-FLAG M2 antibodies (Sigma), anti-HA antibodies (Covance, Princeton, NJ), anti-VDAC1 antibodies (ab14734, Abcam, Cambridge, UK), anti-GST (B-14, Santa Cruz Biotechnology, Santa Cruz, CA), anti-PARP, anti-GAPDH (Cell Signaling Technology [CST], Danvers, MA) and anti-HSP90 (BD Transduction Laboratories, San Diego, CA).

\subsection{Lentiviral vector production}

We produced lentiviruses according to the manufacturer's instructions by cotransfecting the plasmid pLEX-MCS (Thermo Scientific, Rockford, IL), along with the helper plasmids pCAGGS-VSV-G, pCAG4-RTR2 and pCAGkGP1.1R, into HEK293T cells using Fugene HD reagent according to the manufacturer's instructions. Three days after transfection, the culture supernatant was collected and then the cells were infected with the viral supernatant.

\subsection{In vivo ubiquitination assay}


Cell lysates were heated at $90^{\circ} \mathrm{C}$ for $10 \mathrm{~min}$ in $1 \% \mathrm{SDS}$ to remove non-covalent associates, followed by ten-fold dilution with a buffer containing $1 \%$ Triton $\mathrm{X}-100,50$ $\mathrm{mM}$ Tris- $\mathrm{HCl} \mathrm{pH} 7.9$ and $150 \mathrm{mM} \mathrm{NaCl}$ and then immunoprecipitation with $\mathrm{M} 2$ agarose. The agarose was washed five times with a lysis buffer containing $1 \%$ Triton X-100 and $0.1 \%$ SDS. Immune complexes were detected with an anti-FLAG or HA antibody.

\subsection{In vitro ubiquitination assay}

In vitro ubiquitination assays were performed as previously described [31, 32]. An expression vector encoding HA-VDAC1 was transfected into HEK293T cells by FugeneHD reagent. Thirty-six hours after transfection, the cells were collected and lysed in a lysis buffer. HA-VDAC1 was immunoprecipitated with anti-HA-affinity gel (Sigma), and the immunocomplexes were washed five times with the lysis buffer. HA-beads were then incubated at $30^{\circ} \mathrm{C}$ for $2 \mathrm{~h}$ with gentle agitation in the presence of the following components: HEPES-NaOH (pH 7.9), $5 \mathrm{mM} \mathrm{MgCl}_{2}, 60 \mathrm{mM}$ potassium acetate, $1 \mathrm{mM}$ ATP, $2 \mathrm{mM}$ DTT, $0.5 \mathrm{mM}$ EDTA, $100 \mathrm{ng}$ of recombinant E1 (Boston Biochem, Cambridge, MA), 500 ng of recombinant E2 (UbcH1, UbcH2, UbcH3, UbcH5A, UbcH5B, UbcH5C, UbcH6, UbcH7, UbcH8, Ubc2A, Ubc2B, Ubc13/Uev1a or UbcH2S: Boston Biochem) and $5 \mu \mathrm{g}$ of His-ubiquitin with or without recombinant FLAG-RNF207(WT). The reaction was terminated by the addition of an SDS sample buffer containing $4 \% \beta$-mercaptoethanol and heating at $95^{\circ} \mathrm{C}$ for $5 \mathrm{~min}$. Samples were subjected to Western blot analysis with anti-HA antibody. 


\subsection{Immunoprecipitation and affinity purification for proteomic analysis}

We purified protein complexes from cells stably or transiently expressing FLAG-tagged proteins using anti-FLAG M2 agarose as described previously [33].

\subsection{Quantitative real-time PCR}

Total RNA was isolated with the use of Isogen II (Nippon Gene, Tokyo). For reverse transcription-quantitative PCR (RT-qPCR), we reverse-transcribed total mRNA using the iScript Select cDNA Synthesis Kit (Bio-Rad, Hercules, CA), and we performed real-time PCR reactions using an Applied Biosystems StepOne Realtime PCR System with Power SYBR Green PCR Master Mix (Applied Biosystems, Foster City, CA) or an Applied Biosystems 7300 real-time PCR system with TaqMan Gene Expression Master Mix (Applied Biosystems). The cycle threshold (Ct) values were normalized to Gapdh or $18 S$ rRNA. The following primers were used for the RT-qPCR: rat GAPDH forward (Fw) 5'-ACTCCCATTCTTCCACCTTTG-3', rat GAPDH reverse

(Rev) 5'-CCCTGTTGCTGTAGCCATATT-3', rat RNF207 (Fw) 5'GCTACCCAGCAGGAGATTTATG-3', rat RNF207 (Rev) 5'CTTGGTGATGGTGGTGAGATAG-3', rat mitochondrial D-loop (Fw) 5'-GGTTCTTACTTCAGGGCCATCA-3', rat mitochondrial D-loop (Rev) 5'-GATTAGACCCGTTACCATCGAGAT-3', $\quad$ rat $\quad \beta$-actin $\quad$ (Fw) 5'-GGGATGTTTGCTCCAACCAA-3', rat $\quad \beta$-actin $\quad$ (Rev) 5'-GCGCTTTTGACTCAAGGATTTAA-3', mouse RNF207 (Applied Biosystems, 
Mm01260267_g1) and eukaryotic 18S rRNA endogenous control (Applied Biosystems).

\subsection{5. siRNA transfection}

We transfected rat neonatal cardiomyocytes in six-well tissue culture plates

$\left(\sim 1 \times 10^{6}\right.$ cells $/$ well $)$ or in 6 -cm dishes $\left(\sim 3 \times 10^{6}\right.$ cells $/$ dish $)$ or 10 -cm dishes $\left(\sim 6 \times 10^{6}\right.$ cells/dish) with $50 \mathrm{nM}$ RNF207 siRNA (\#1-3) or with $50 \mathrm{nM}$ negative control siRNA (\#1-3) using Lipofectamine ${ }^{\mathrm{TM}}$ RNAiMAX Transfection Reagent (Invitrogen). RNF207 siRNA\#1 and negative control siRNA\#1 were obtained from Ambion (Life Technologies, Carlsbad, CA). RNF207 siRNA\#2 and negative control siRNA\#2 were obtained from Dharmacon (GE Healthcare). RNF207 siRNA\#3 and negative control siRNA\#3 were obtained from Sigma.

\subsection{Metabolomic analysis}

The culture medium was aspirated from the dish and the cells were then washed twice with 5\% mannitol solution (10 $\mathrm{ml}$ first and then $2 \mathrm{ml})$. The cells were then treated with $800 \mu \mathrm{l}$ of methanol and incubated for $30 \mathrm{~s}$ to inactivate enzymes. Next, the cell extract was treated with $550 \mu \mathrm{l}$ of Milli-Q water containing internal standards (H3304-1002, Human Metabolome Technologies, Tsuruoka, Japan) and incubated for a further $30 \mathrm{~s}$. The extract was then collected and centrifuged at $2,300 \mathrm{~g}$ for $5 \mathrm{~min}$ at $4^{\circ} \mathrm{C}$, and then $800 \mu 1$ of the upper aqueous layer was centrifugally filtered through a Millipore 5-kDa cutoff filter at $9,100 \mathrm{~g}$ for $120 \mathrm{~min}$ at $4^{\circ} \mathrm{C}$ to remove proteins. The concentrated samples were resuspended in $50 \mu \mathrm{l}$ of Milli-Q water for capillary 
electrophoresis-mass spectrometry (CE-MS) analysis. Each measurement of metabolites was normalized to total protein.

\subsection{Measurement of mitochondrial respiration}

Mitochondrial respiration was measured using permeabilized rat cardiomyocytes as previously described [34]. Complex I-linked state 3 respiration was assessed with malate $(5 \mathrm{mM})$, glutamate $(10 \mathrm{mM})$ and a saturating concentration of ADP $(5 \mathrm{mM})$.

Subsequently, oligomycin $\left(2 \mu \mathrm{g} \mathrm{ml}^{-1}\right)$ was added to inhibit ATP synthase. This was followed by FCCP $(2.5 \mu \mathrm{M})$ to assess the maximum $\mathrm{O}_{2}$ consumption rate (OCR). Finally, antimycin A $(2.5 \mu \mathrm{M})$ was added to inhibit complex III, and non-mitochondrial respiration was determined. Absolute respiration values were normalized to the total number of cells per chamber.

\subsection{Measurement of mitochondrial membrane potential}

We measured mitochondrial membrane potential with a JC-10 Mitochondrial Membrane Potential Kit (Abcam, Cambridge, MA) according to the manufacturer's instructions. This assay was performed at $96 \mathrm{~h}$ after siRNA transfection. The mitochondrial membrane potential is expressed as a ratio ( $\mathrm{J}$ aggregates/monomer).

\subsection{Measurement of mitochondrial DNA (mtDNA) copy number}

We extracted DNA from rat neonatal cardiomyocytes with a QIAamp DNA Mini 
Kit (Qiagen, Westburg, The Netherlands) according to the manufacturer's instructions. The cycle number differences between the primers for a nuclear gene ( $\beta$-actin) and a mitochondrial genome (D-loop) were measured in qPCR reactions as previously described [35].

2.20. Measurement of pyruvate dehydrogenase activity in cardiomyocytes

We measured pyruvate dehydrogenase activity with a Pyruvate Dehydrogenase (PDH) Enzyme Activity Microplate Assay Kit (ab109902, Abcam) according to the manufacturer's instructions. Each measurement was normalized to the total protein.

\subsection{Statistical analysis}

We used the unpaired Student's $t$ test to determine the statistical significance of the experimental data. 


\section{Results}

\subsection{RNF207 is specifically expressed in the heart}

We performed RT-qPCR using cDNAs derived from various tissues of mice, and we observed a predominant expression of Rnf 207 mRNA in the heart and little expression in other tissues (Fig. 1A). To examine the expression of RNF207 at the protein level, we generated an anti-RNF207 antibody by immunization of recombinant mouse RNF207 protein into rabbits and performed Western blot analysis using mouse tissues. The results obtained from Western blot analysis showed that the antibody recognized mouse RNF207 exogenously expressed in HEK293T cells and endogenous RNF207 in NRCs, indicating that the generated antibody is specific to mouse and rat RNF207 (Fig. 2A, Supplemental Fig. 1). Consistent with the results obtained from RT-qPCR, Western blot analysis using several mouse tissues showed that RNF207 was predominantly expressed in the heart, suggesting that RNF207 is associated with cardiac function (Fig. 1B).

3.2. RNF207 is required for maintenance of normal energy metabolism and mitochondrial function in rat neonatal cardiomyocytes

Since the heart continuously contracts to pump blood through the body and the rates of ATP turnover in the heart are larger than those of any other organs [36], we hypothesized that RNF207 is associated with cardiac metabolism. To investigate this hypothesis, we transfected siRNA targeting RNF207 into NRCs and performed 
metabolomic analysis to determine the amounts of various intracellular metabolites. As expected, the results obtained from metabolomic analysis showed that the ATP concentration and the $\mathrm{NADH} / \mathrm{NAD}^{+}$ratio were significantly lower in NRCs treated with siRNA targeting RNF207 than in NRCs treated with non-targeting siRNA (Fig. 2A-C).

In addition, RNF207 knockdown moderately decreased the level of tricarboxylic cycle metabolites in NRCs (Supplemental Fig. 2A), although there was no significant difference between the RNF207-depleted NRCs and control NRCs. Based on these results, we hypothesized that mitochondrial respiratory function was impaired in RNF207-depleted NRCs. To directly evaluate mitochondrial respiratory function, we measured the cellular maximal $\mathrm{O}_{2}$ consumption rate (OCR). The maximal OCR was significantly reduced in RNF207-depleted NRCs compared to that in non-treated NRCs (Fig. 2D). In addition, mitochondrial membrane potential was significantly reduced in RNF207-depleted NRCs compared to that in non-treated NRCs (Supplemental Fig. 2B). The reduced oxidative phosphorylation observed in RNF207-depleted NRCs was not caused by altered numbers of mitochondria, since the amounts of mitochondrial DNA were similar in the two types of cardiomyocytes (Supplemental Fig. 2C). In contrast, RNF207 knockdown had no effect on metabolites associated with the glycolysis pathway in NRCs (Supplemental Fig. 2D).

In light of these results, we speculated that the activity of an enzyme linking glycolysis to oxidative phosphorylation was reduced. We therefore measured the activity of pyruvate dehydrogenase (PDH), which converts pyruvate to acetyl-CoA. As expected, PDH activity was significantly reduced in RNF207-depleted NRCs compared to that in control NRCs (Fig. 2E). In addition, we found that transfection of NRCs with siRNAs targeting RNF207 induced a decrease in cell size, especially when the 
confluency of the NRCs was relatively low (Fig. 2F-G). Although we investigated the possibility that depletion of RNF207 caused apoptosis, there was no induction of apoptosis in RNF207-depleted NRCs (Supplemental Fig. 2E-F).

Intriguingly, the metabolomic analysis also showed that the amounts of almost all amino acids were significantly decreased in RNF207 knocked-down NRCs (Fig. 2H). These findings suggest that RNF207 plays an important role in the regulation of energy metabolism in cardiomyocytes.

\subsection{RNF207 directly interacts with VDAC1}

To elucidate the molecular mechanism by which RNF207 affects cardiac energy metabolism, we performed mass spectrometry of RNF207-interacting proteins using FLAG-tagged RNF207 (FLAG-RNF207). We exogenously expressed the $3 \times$ FLAG-RNF207 wild type (WT) and 3×FLAG-RNF207 RING-finger domain deletion mutant $(\Delta \mathrm{R})$ in HEK293T cells, and we expressed FLAG-RNF207 (WT) in NRCs. We also generated mouse myoblast $\mathrm{C} 2 \mathrm{C} 12$ cells stably expressing $3 \times$ FLAG-RNF207 (WT). As shown in Fig. 3A and B, both RNF207 WT and $\Delta$ R copurified with a protein, the apparent molecular mass of which was approximately 35,000 (35-kDa protein) (Fig. 3A, B, Supplemental Fig. 3A).

The mass spectrometric analysis revealed that the $35-\mathrm{kDa}$ protein is a mitochondrial outer membrane porin, VDAC1 (Supplemental Fig. 3B). Although VDAC2 was also detected by mass spectrometry, the amino acid sequence coverage rate of VDAC2 was lower than that of VDAC1 (data not shown). It has been shown that VDAC is one of the most abundant proteins in the outer mitochondrial membrane and is an important 
regulator of mitochondrial metabolism [37]. Since it has been reported that cardiomyocytes contain a large amount of mitochondria, constituting $30 \%-40 \%$ of cellular volume [38], we hypothesized that RNF207 plays an important role in mitochondria in collaboration with VDAC. Since it has been reported that VDAC1 is a predominant isoform in many tissues [39], we focused on VDAC1. Interaction between RNF207 and VDAC1 was confirmed by the results of our co-precipitation experiments. Endogenous VDAC1 was co-precipitated with FLAG or $3 \times$ FLAG-RNF207 (WT) in human, rat and mouse cells (Fig. 3C, D, Supplemental Fig. 3C).

To rule out the possibility that RNF207 interacted with endogenous VDAC1 through proteins such as a chaperone induced by the overexpression of RNF207, we generated recombinant FLAG-RNF207 (WT) using a baculoviral expression system and tested whether recombinant FLAG-RNF207 copurifies with VDAC1 from mouse heart lysates. As shown in Fig. 3E, recombinant FLAG-RNF207 (WT) copurified with VDAC1 from mouse heart lysates.

To investigate the possibility of a direct interaction between RNF207 and VDAC1, we performed in vitro binding assays. We generated bacterially expressed recombinant glutathione $S$-transferase (GST)-tagged VDAC1 (GST-VDAC1) and FLAG-RNF207 $(\Delta \mathrm{R})$, and then we incubated FLAG-RNF207 $(\Delta \mathrm{R})$ pre-bound to M2 agarose with GST-VDAC1. Western blot analysis showed that recombinant FLAG-RNF207 $(\Delta \mathrm{R})$ was co-precipitated with recombinant GST-VDAC1 but not with GST alone (Fig. 3F). These findings suggest that RNF207 directly interacts with VDAC1.

\subsection{RNF207 is associated with VDAC1 through the coiled-coil region}


RNF207 is structurally similar to tripartite motif (TRIM) proteins. TRIM proteins are characterized by the presence of a zinc-binding RING finger, one or two zinc-binding motifs called B-boxes, an associated coiled-coil region called the B-box C-terminal domain (BBC) and unique carboxyl-terminal domains [40]. It has been reported that the region containing B-boxes and the coiled-coil is likely to be responsible for the interaction with substrates [41].

To clarify the region of RNF207 required for binding to VDAC1, we performed binding assays using several deletion mutants of RNF207. We expressed a series of $3 \times$ FLAG-tagged RNF207 deletion mutants including RNF207 $(\Delta \mathrm{R})$, RNF207 $(\Delta \mathrm{B})$, RNF207 (RB), RNF207 ( $\triangle$ RB) and RNF207 ( $\triangle$ RBBC) in HEK293T cells. Western blot analysis of co-precipitates with M2 agarose revealed that endogenous VDAC1 was co-precipitated with RNF207 $(\Delta \mathrm{R})$, RNF207 ( $\Delta \mathrm{B})$ and RNF207 ( $\Delta$ RB) but not with RNF207 (RB) or RNF207 (ARBBC) (Fig. 4A, B).

We further performed co-precipitation experiments using three types of $3 \times$ FLAG-tagged RNF207 coiled-coil region mutants, BBC1, BBC2 and BBC3. The results of Western blot analysis showed that $\mathrm{BBC} 1$ and $\mathrm{BBC} 3$ interacted with endogenous VDAC1 but that BBC2 did not (Fig. 4A, C). These findings suggest that the region from 263-302 amino acid residues in the coiled-coil region is required for the interaction between RNF207 and VDAC1. We also confirmed that an RNF207-truncated mutant in which the region from 263 to 302 amino acid residues was deleted did not interact with VDAC1 (Supplemental Fig. 4).

We next found by multiple amino acid sequence alignments that this region is highly conserved among vertebrate species (Fig. 4D). Among the highly conserved amino acids, we focused on seven hydrophilic amino acids that were presumed to be 
molecular surface residues. We generated three expression vectors encoding $3 \times$ FLAG-tagged BBC3 point mutants in which two or three hydrophilic residues were substituted with alanine: BBC3 (EEK270-272AAA), BBC3 (DK273-274AA) and BBC3 (KE277-278AA) (Fig. 4D). Our in vivo binding assays using those three FLAG-BBC3 point mutants showed that BBC3 (KE277-278AA) interacted much more weakly with endogenous VDAC1 than did BBC3 (EEK270-272AAA) or BBC3 (DK273-274AA), suggesting that lysine 277 and glutamate 278 residues are critical for the interaction of RNF207 with VDAC1 (Fig. 4E).

It has been reported that the N-terminal domain of VDAC1 is important for its interaction with various proteins, especially cytosolic proteins including hexokinase1 and $\mathrm{Bcl} 2$ family proteins [42]. To clarify the region of VDAC1 that is required for interaction with RNF207, we performed co-precipitation experiments using full-length VDAC1 and a VDAC1-N-terminal truncate $(\Delta 28)$. We found that HA-RNF207 (WT) interacts with full-length VDAC1 much more strongly than it does with VDAC1 $(\Delta 28)$, suggesting that the N-terminal domain of VDAC1 is required for the interaction with RNF207 (Fig. 4F).

\subsection{RNF207 promotes ubiquitination of VDAC1}

RNF207 has a RING-finger domain, which is known to be required for the activities of E3 ubiquitin ligases [43]. To examine whether RNF207 ubiquitinates VDAC1, we performed in vivo ubiquitination assays. We found that RNF207 overexpression promotes ubiquitination of VDAC1 (Supplemental Fig. 5A). We next examined whether RNF207 ubiquitinates VDAC1 in vitro. We purified HA-VDAC1 
using HA-agarose from lysates of HEK293T cells exogenously expressing HA-VDAC1 and then performed an in vitro ubiquitination assay using HA-VDAC1 bound to HA agarose as a substrate. We found that RNF207 (WT) enhances ubiquitination of VDAC1 (Supplemental Fig. 5B). These findings suggest that RNF207 is involved in the ubiquitination of VDAC1. To examine whether RNF207 plays a role in proteasomal degradation of VDAC1, we checked the stability of VDAC1 in the presence of cycloheximide, an inhibitor of protein synthesis. We actually examined whether exogenous expression of RNF207 or knockdown of RNF207 affects the expression levels of endogenous VDAC1 in HEK293T cells or NRCs. We found that neither exogenous RNF207 expression nor knockdown of RNF207 affected the stability of endogenous VDAC1 (Supplemental Fig. 5C, D), suggesting that RNF207 is not involved in the proteasomal degradation of VDAC1.

Since it has been reported that the oligomerization of VDAC may play an important role in the interactions between VDAC and associated proteins such as hexokinase and mitochondrial creatine kinase [44], we performed a sucrose-density gradient centrifugation assay to examine whether RNF207 affects the formation of a VDAC1 oligomerization state. However, knockdown of RNF207 in NRCs had no effect on the oligomerization state of VDAC1 (Supplemental Fig. 5E).

\subsection{Several stress stimuli attenuate the expression of RNF207 in the heart}

To clarify the function of RNF207 in common cardiac diseases, we investigated whether the expression of RNF207 changed in mouse models of cardiac disease, including a transverse aortic constriction (TAC) model, an ischemia/reperfusion (I/R) 
model and a coronary ischemic HF model (Supplemental Tables 1 and 2, Supplemental Fig. 6). We found that at 4 weeks after TAC and at $24 \mathrm{~h}$ after reperfusion, the mRNA level of RNF207 in the hearts was significantly decreased to approximately $40 \%$ of that in sham mice (Fig. 5A, B). Moreover, we found that the protein level of RNF207 in the hearts with HF was significantly reduced to approximately $70 \%$ of that in sham mice (Fig. 5C, D). These findings suggest that the expression level of RNF207 is involved in the progression of cardiac hypertrophy, ischemic heart disease and HF.

Since it has been reported that TAC causes oxidative stress and ER stress $[45,46]$ and that $\mathrm{I} / \mathrm{R}$ causes oxidative stress, ER stress and calcium overload [47, 48], we hypothesized that several stress stimuli cause a change in the expression of RNF207. To examine this hypothesis, we incubated NRCs with phenylephrine, hydrogen peroxide, tunicamycin (TM) and A23187, which induce hypertrophic stress, oxidative stress, ER stress and calcium overload, respectively. RT-qPCR analysis showed that these stress inducers significantly reduced the mRNA levels of RNF207 in NRCs (Fig. 5E-H). We also confirmed that the expression of RNF207 protein was significantly decreased in NRCs exposed to TM (Fig. 5I).

Intriguingly, we also found that glucose deprivation, which induces ER stress, oxidative stress and other types of stress [49], attenuated the interaction between RNF207 and VDAC1 (Fig. 5J). These results may indicate that RNF207 functions as a stress-responsive sensor in hearts. 


\section{Discussion}

We obtained evidence that RNF207 is a novel heart-specific protein and that it interacts with VDAC1. Consistent with the results of studies showing that RNF207 is highly expressed only in the heart at the mRNA level $[20,50]$, our findings revealed that RNF207 is predominantly expressed in the heart at both the mRNA and protein levels. Given the cardiac specificity of RNF207, RNF207 may be a good candidate for a cardiac marker in several heart diseases. In fact, it has been reported that RNF207 in patients with acute myocardial infarction (AMI) exhibited sensitivity and specificity equal to those of cardiac-specific troponin I, which is used worldwide for the diagnosis of AMI [50]. In addition, since it has been reported that cardiac-specific troponin $\mathrm{T}$ is helpful for risk stratification in acutely decompensated HF [51], RNF207 may serve as a prognostic marker in HF patients.

Our findings also demonstrated that RNF207 is associated with energy metabolism in isolated cardiomyocytes. However, the precise molecular mechanism by which RNF207 affected the energy metabolism in isolated cardiomyocytes is not clear. Recent studies have shown that genetic variation in RNF207 is associated with prolongation of the QT interval $[52,53]$ and that RNF207 knockdown in zebrafish embryos prolonged the action potential duration (APD) [21]. It was also reported that VDAC1 is the only identified protein mediating $\mathrm{Ca}^{2+}$ transport in the outer mitochondrial membrane [54] and that $\mathrm{Ca}^{2+}$ dynamics is important not only for APD $[55,56]$ but also for the regulation of cellular energetic production [57-59]. These findings raised the possibility that RNF207 regulates cellular $\mathrm{Ca}^{2+}$ dynamics, especially the mitochondrial $\mathrm{Ca}^{2+}$ concentration. We attempted to measure the $\mathrm{Ca}^{2+}$ concentration in the mitochondria of 
isolated cardiomyocytes, but we did not observe a significant difference between NRCs treated with siRNA targeting RNF207 and NRCs treated with non-targeting siRNA (data not shown). Therefore, further experiments may be needed to address this question.

Our observation that the interaction between RNF207 and VDAC1 was disrupted by glucose starvation, which changes cellular metabolism, may indicate that interaction between RNF207 and VDAC1 is associated with the regulation of cellular metabolism. Actually, a previous study showed that VDAC is involved in cardiac energy metabolism [60]. Although we demonstrated that RNF207 promotes the polyubiquitination of VDAC1, RNF207 does not affect the stability of VDAC1. The mechanisms by which RNF207 regulates VDAC1 function through ubiquitination remain to be elucidated. Further study is needed to clarify how the interaction of RNF207 with VDAC1 is associated with cardiac energy metabolism.

We also showed that the expression of RNF207 was reduced by various stimuli that induce hypertrophic stress, oxidative stress, ER stress and calcium overload in vivo and vitro. Consistent with our results, the results of microarray analyses available from the NCBI public functional genomics data repository $[21,61]$ showed that the mRNA level of Rnf207 is significantly reduced in NRCs treated with the pyridine activator of myocyte hypertrophy, which induces cardiac hypertrophy [62], and in mouse hearts treated with isoproterenol, which causes tachycardia-induced HF [63].

It has also been shown that cardiac energy metabolism changes and the levels of ATP in cardiomyocytes are reduced in three different mouse models of cardiac hypertrophy, cardiac ischemia and chronic $\operatorname{HF}[3,64,65]$. In the present study, we found that RNF207 knockdown had an effect on energy metabolism in isolated 
cardiomyocytes and that the expression level of $R n f 207$ was reduced in mouse model hearts, supporting the possibility that the downregulation of RNF207 expression plays an important role in cardiac metabolic change and the reduction of ATP production in the heart. Considering a previous study demonstrating that mouse hearts that underwent ischemic preconditioning showed lower ATP concentrations than those in control hearts [66], the reduction of RNF207 expression at an early stage may have a protective effect against ischemic injury. However, continuous reduction of RNF207 expression probably has a detrimental effect on HF. Although chronic HF is a multifactorial disease, altered energy metabolism has been suggested as one of the most important causes of HF [3]. In fact, several studies have shown that interventions for cardiac metabolic change improve cardiac function and prevent the progression of cardiac dysfunction [67-69]. Elucidation of the mechanisms by which RNF207 plays a role in energy metabolism in HF may uncover new therapeutic strategies for HF. 


\section{Acknowledgments}

We would like to thank Dr. Arthur Nienhuis (St. Jude Children's Research Hospital) for kindly providing pCAGGS-VSV-G plasmid, pCAGkGP1.1R plasmid and pCAG4-RTR2 plasmid, Dr. Masahiko Watanabe (Hokkaido University) for support in generating an anti-RNF207 antibody, Ms. Yuri Soida and Ms. Miho Uchiumi for their helping in preparing the manuscript, Ms. Misumi Matsuo for technical assistance and Ms. Mizuho Oda for help in performing the mass spectrometric analysis.

This work was supported in part by KAKENHI $(24112006,15 \mathrm{H} 04690)$ from the Ministry of Education, Culture, Sports, Science and Technology in Japan and by Japan Diabetes Foundation and the Uehara Memorial Foundation (to S. Hatakeyama).

\section{Conflict of interest}

None. 


\section{References}

[1] S. Stewart, K. MacIntyre, S. Capewell, J.J. McMurray, Heart failure and the aging population: an increasing burden in the 21st century?, Heart 89 (2003) 49-53.

[2] Y. Sata, H. Krum, The future of pharmacological therapy for heart failure, Circ. J. 74 (2010) 809-817.

[3] S. Neubauer, The failing heart--an engine out of fuel, N. Engl. J. Med. 356 (2007) 1140-1151.

[4] S.C. Kolwicz, Jr., S. Purohit, R. Tian, Cardiac metabolism and its interactions with contraction, growth, and survival of cardiomyocytes, Circ. Res. 113 (2013) 603-616.

[5] P.S. Azevedo, M.F. Minicucci, P.P. Santos, S.A. Paiva, L.A. Zornoff, Energy metabolism in cardiac remodeling and heart failure, Cardiol. Rev. 21 (2013) 135-140.

[6] J.M. Huss, D.P. Kelly, Nuclear receptor signaling and cardiac energetics, Circ. Res. 95 (2004) 568-578.

[7] T. Doenst, T.D. Nguyen, E.D. Abel, Cardiac metabolism in heart failure: implications beyond ATP production, Circ. Res. 113 (2013) 709-724.

[8] G. Aubert, O.J. Martin, J.L. Horton, L. Lai, R.B. Vega, T.C. Leone, et al., The Failing Heart Relies on Ketone Bodies as a Fuel, Circulation 133 (2016) 698-705.

[9] H.A. Yoshihara, J.A. Bastiaansen, C. Berthonneche, A. Comment, J. Schwitter, An intact small animal model of myocardial ischemia-reperfusion: Characterization of metabolic changes by hyperpolarized 13C MR spectroscopy, Am. J. Physiol. Heart Circ. Physiol. 309 (2015) H2058-2066.

[10] P.S. Freemont, I.M. Hanson, J. Trowsdale, A novel cysteine-rich sequence motif, 
Cell 64 (1991) 483-484.

[11] Y. Yang, S. Fang, J.P. Jensen, A.M. Weissman, J.D. Ashwell, Ubiquitin protein ligase activity of IAPs and their degradation in proteasomes in response to apoptotic stimuli, Science 288 (2000) 874-877.

[12] H. Duan, Y. Wang, M. Aviram, M. Swaroop, J.A. Loo, J. Bian, et al., SAG, a novel zinc RING finger protein that protects cells from apoptosis induced by redox agents, Mol. Cell. Biol. 19 (1999) 3145-3155.

[13] J.H. Lee, G.Y. Lee, H. Jang, S.S. Choe, S.H. Koo, J.B. Kim, Ring finger protein20 regulates hepatic lipid metabolism through protein kinase A-dependent sterol regulatory element binding protein1c degradation, Hepatology 60 (2014) 844-857.

[14] B.K. Koo, M. Spit, I. Jordens, T.Y. Low, D.E. Stange, M. van de Wetering, et al., Tumour suppressor RNF43 is a stem-cell E3 ligase that induces endocytosis of Wnt receptors, Nature 488 (2012) 665-669.

[15] G.S. Stewart, S. Panier, K. Townsend, A.K. Al-Hakim, N.K. Kolas, E.S. Miller, et al., The RIDDLE syndrome protein mediates a ubiquitin-dependent signaling cascade at sites of DNA damage, Cell 136 (2009) 420-434.

[16] W. Liu, D. Morito, S. Takashima, Y. Mineharu, H. Kobayashi, T. Hitomi, et al., Identification of RNF213 as a susceptibility gene for moyamoya disease and its possible role in vascular development, PLoS One 6 (2011) e22542.

[17] Y. Zhang, Y.M. Kang, C. Tian, Y. Zeng, L.X. Jia, X. Ma, et al., Overexpression of Nrdp1 in the heart exacerbates doxorubicin-induced cardiac dysfunction in mice, PLoS One 6 (2011) e21104.

[18] D. Gero, P. Szoleczky, A. Chatzianastasiou, A. Papapetropoulos, C. Szabo, Modulation of poly(ADP-ribose) polymerase-1 (PARP-1)-mediated oxidative cell 
injury by ring finger protein 146 (RNF146) in cardiac myocytes, Mol. Med. 20 (2014) 313-328.

[19] Y.Q. Liu, D.C. Tao, S.Y. Liao, Y. Yang, Y.X. Ma, S.Z. Zhang, Identification of a novel human testicular interstitial gene, RNF148, and its expression regulated by histone deacetylases, Genet. Mol. Res. 12 (2013) 4060-4069.

[20] S. Uchida, A. Schneider, M. Wiesnet, B. Jungblut, P. Zarjitskaya, K. Jenniches, et al., An integrated approach for the systematic identification and characterization of heart-enriched genes with unknown functions, BMC genomics 10 (2009) 100.

[21] K. Roder, A.A. Werdich, W. Li, M. Liu, T.Y. Kim, L.E. Organ-Darling, et al., RING finger protein RNF207, a novel regulator of cardiac excitation, J. Biol. Chem. 289 (2014) 33730-33740.

[22] S.J. Schein, M. Colombini, A. Finkelstein, Reconstitution in planar lipid bilayers of a voltage-dependent anion-selective channel obtained from paramecium mitochondria, J. Membr. Biol. 30 (1976) 99-120.

[23] V. Shoshan-Barmatz, D. Mizrachi, VDAC1: from structure to cancer therapy, Front. Oncol. 2 (2012) 164.

[24] Y. Sun, A.A. Vashisht, J. Tchieu, J.A. Wohlschlegel, L. Dreier, Voltage-dependent anion channels (VDACs) recruit Parkin to defective mitochondria to promote mitochondrial autophagy, J. Biol. Chem. 287 (2012) 40652-40660.

[25] H. Huang, X. Hu, C.O. Eno, G. Zhao, C. Li, C. White, An interaction between Bcl-xL and the voltage-dependent anion channel (VDAC) promotes mitochondrial Ca2+ uptake, J. Biol. Chem. 288 (2013) 19870-19881.

[26] T. Sato, H. Takahashi, S. Hatakeyama, A. Iguchi, T. Ariga, The TRIM-FLMN protein TRIM45 directly interacts with RACK1 and negatively regulates 
PKC-mediated signaling pathway, Oncogene 34 (2015) 1280-1291.

[27] S. Matsushima, J. Kuroda, T. Ago, P. Zhai, J.Y. Park, L.H. Xie, et al., Increased oxidative stress in the nucleus caused by Nox4 mediates oxidation of HDAC4 and cardiac hypertrophy, Circ. Res. 112 (2013) 651-663.

[28] A. Yamamoto, R. Takeya, M. Matsumoto, K.I. Nakayama, H. Sumimoto, Phosphorylation of Noxo1 at threonine 341 regulates its interaction with Noxa1 and the superoxide-producing activity of Nox1, FEBS J. 280 (2013) 5145-5159.

[29] H. Takahashi, T.J. Parmely, S. Sato, C. Tomomori-Sato, C.A. Banks, S.E. Kong, et al., Human mediator subunit MED26 functions as a docking site for transcription elongation factors, Cell 146 (2011) 92-104.

[30] H. Takahashi, S. Hatakeyama, H. Saitoh, K.I. Nakayama, Noncovalent SUMO-1 binding activity of thymine DNA glycosylase (TDG) is required for its SUMO-1 modification and colocalization with the promyelocytic leukemia protein, J. Biol. Chem. 280 (2005) 5611-5621.

[31] T. Kamura, T. Hara, M. Matsumoto, N. Ishida, F. Okumura, S. Hatakeyama, et al., Cytoplasmic ubiquitin ligase KPC regulates proteolysis of p27(Kip1) at G1 phase, Nat. Cell Biol. 6 (2004) 1229-1235.

[32] A. Peschiaroli, N.V. Dorrello, D. Guardavaccaro, M. Venere, T. Halazonetis, N.E. Sherman, et al., SCFbetaTrCP-mediated degradation of Claspin regulates recovery from the DNA replication checkpoint response, Mol. Cell 23 (2006) 319-329.

[33] H. Takahashi, S. Martin-Brown, M.P. Washburn, L. Florens, J.W. Conaway, R.C. Conaway, Proteomics reveals a physical and functional link between hepatocyte nuclear factor 4alpha and transcription factor IID, J. Biol. Chem. 284 (2009) 32405-32412. 
[34] M. Gram, A. Vigelso, T. Yokota, C.N. Hansen, J.W. Helge, M. Hey-Mogensen, et al., Two weeks of one-leg immobilization decreases skeletal muscle respiratory capacity equally in young and elderly men, Exp. Gerontol. 58 (2014) 269-278.

[35] J.A. Nicklas, E.M. Brooks, T.C. Hunter, R. Single, R.F. Branda, Development of a quantitative PCR (TaqMan) assay for relative mitochondrial DNA copy number and the common mitochondrial DNA deletion in the rat, Environ Mol Mutagen 44 (2004) 313-320.

[36] H. Taegtmeyer, Energy metabolism of the heart: from basic concepts to clinical applications, Curr. Probl. Cardiol. 19 (1994) 59-113.

[37] M. Colombini, VDAC: the channel at the interface between mitochondria and the cytosol, Mol. Cell. Biochem. 256-257 (2004) 107-115.

[38] F. Giorgianni, D. Koirala, S. Beranova-Giorgianni, Proteomics of the human pituitary tissue: bioanalytical methods and applications, Bioanalysis 6 (2014) 1989-2003.

[39] V. Shoshan-Barmatz, V. De Pinto, M. Zweckstetter, Z. Raviv, N. Keinan, N. Arbel, VDAC, a multi-functional mitochondrial protein regulating cell life and death, Mol. Aspects Med. 31 (2010) 227-285.

[40] S. Hatakeyama, TRIM proteins and cancer, Nat. Rev. Cancer 11 (2011) 792-804.

[41] G. Meroni, G. Diez-Roux, TRIM/RBCC, a novel class of 'single protein RING finger' E3 ubiquitin ligases, Bioessays 27 (2005) 1147-1157.

[42] S. Abu-Hamad, N. Arbel, D. Calo, L. Arzoine, A. Israelson, N. Keinan, et al., The VDAC1 N-terminus is essential both for apoptosis and the protective effect of anti-apoptotic proteins, J. Cell Sci. 122 (2009) 1906-1916.

[43] R.J. Deshaies, C.A. Joazeiro, RING domain E3 ubiquitin ligases, Annu Rev 
Biochem 78 (2009) 399-434.

[44] R. Zalk, A. Israelson, E.S. Garty, H. Azoulay-Zohar, V. Shoshan-Barmatz, Oligomeric states of the voltage-dependent anion channel and cytochrome c release from mitochondria, Biochem. J. 386 (2005) 73-83.

[45] Z. Lu, X. Xu, X. Hu, S. Lee, J.H. Traverse, G. Zhu, et al., Oxidative stress regulates left ventricular PDE5 expression in the failing heart, Circulation 121 (2010) 1474-1483.

[46] K. Okada, T. Minamino, Y. Tsukamoto, Y. Liao, O. Tsukamoto, S. Takashima, et al., Prolonged endoplasmic reticulum stress in hypertrophic and failing heart after aortic constriction: possible contribution of endoplasmic reticulum stress to cardiac myocyte apoptosis, Circulation 110 (2004) 705-712.

[47] H.W. Yung, S. Korolchuk, A.M. Tolkovsky, D.S. Charnock-Jones, G.J. Burton, Endoplasmic reticulum stress exacerbates ischemia-reperfusion-induced apoptosis through attenuation of Akt protein synthesis in human choriocarcinoma cells, FASEB J. 21 (2007) 872-884.

[48] K. Shintani-Ishida, M. Inui, K. Yoshida, Ischemia-reperfusion induces myocardial infarction through mitochondrial $\mathrm{Ca}(2)(+)$ overload, J. Mol. Cell. Cardiol. 53 (2012) 233-239.

[49] R. Palorini, F.P. Cammarata, C. Balestrieri, A. Monestiroli, M. Vasso, C. Gelfi, et al., Glucose starvation induces cell death in K-ras-transformed cells by interfering with the hexosamine biosynthesis pathway and activating the unfolded protein response, Cell Death Dis. 4 (2013) e732.

[50] Q.Y. Han, H.X. Wang, X.H. Liu, C.X. Guo, Q. Hua, X.H. Yu, et al., Circulating E3 ligases are novel and sensitive biomarkers for diagnosis of acute myocardial 
infarction, Clin. Sci. (Lond) 128 (2015) 751-760.

[51] J.L. Januzzi, Jr., G. Filippatos, M. Nieminen, M. Gheorghiade, Troponin elevation in patients with heart failure: on behalf of the third Universal Definition of Myocardial Infarction Global Task Force: Heart Failure Section, Eur. Heart J. 33 (2012) 2265-2271.

[52] A. Pfeufer, S. Sanna, D.E. Arking, M. Muller, V. Gateva, C. Fuchsberger, et al., Common variants at ten loci modulate the QT interval duration in the QTSCD Study, Nat. Genet. 41 (2009) 407-414.

[53] C. Newton-Cheh, M. Eijgelsheim, K.M. Rice, P.I. de Bakker, X. Yin, K. Estrada, et al., Common variants at ten loci influence QT interval duration in the QTGEN Study, Nat. Genet. 41 (2009) 399-406.

[54] D. Gincel, H. Zaid, V. Shoshan-Barmatz, Calcium binding and translocation by the voltage-dependent anion channel: a possible regulatory mechanism in mitochondrial function, Biochem. J. 358 (2001) 147-155.

[55] C.T. January, J.M. Riddle, Early afterdepolarizations: mechanism of induction and block. A role for L-type Ca2+ current, Circ. Res. 64 (1989) 977-990.

[56] I. Itzhaki, L. Maizels, I. Huber, L. Zwi-Dantsis, O. Caspi, A. Winterstern, et al., Modelling the long QT syndrome with induced pluripotent stem cells, Nature 471 (2011) 225-229.

[57] M.J. Bround, R. Wambolt, D.S. Luciani, J.E. Kulpa, B. Rodrigues, R.W. Brownsey, et al., Cardiomyocyte ATP production, metabolic flexibility, and survival require calcium flux through cardiac ryanodine receptors in vivo, J. Biol. Chem. 288 (2013) 18975-18986.

[58] B. Glancy, R.S. Balaban, Role of mitochondrial Ca2+ in the regulation of cellular 
energetics, Biochemistry 51 (2012) 2959-2973.

[59] C. Cardenas, R.A. Miller, I. Smith, T. Bui, J. Molgo, M. Muller, et al., Essential regulation of cell bioenergetics by constitutive InsP3 receptor $\mathrm{Ca} 2+$ transfer to mitochondria, Cell 142 (2010) 270-283.

[60] R. Guzun, T. Kaambre, R. Bagur, A. Grichine, Y. Usson, M. Varikmaa, et al., Modular organization of cardiac energy metabolism: energy conversion, transfer and feedback regulation, Acta Physiol. (Oxf) 213 (2015) 84-106.

[61] R. Edgar, M. Domrachev, A.E. Lash, Gene Expression Omnibus: NCBI gene expression and hybridization array data repository, Nucleic Acids Res. 30 (2002) 207-210.

[62] E.W. Bush, D.B. Hood, P.J. Papst, J.A. Chapo, W. Minobe, M.R. Bristow, et al., Canonical transient receptor potential channels promote cardiomyocyte hypertrophy through activation of calcineurin signaling, J. Biol. Chem. 281 (2006) 33487-33496.

[63] C.L. Galindo, M.A. Skinner, M. Errami, L.D. Olson, D.A. Watson, J. Li, et al., Transcriptional profile of isoproterenol-induced cardiomyopathy and comparison to exercise-induced cardiac hypertrophy and human cardiac failure, BMC Physiol. 9 (2009) 23.

[64] H. Tagashira, C. Zhang, Y.M. Lu, H. Hasegawa, H. Kanai, F. Han, et al., Stimulation of sigma1-receptor restores abnormal mitochondrial $\mathrm{Ca}(2)(+)$ mobilization and ATP production following cardiac hypertrophy, Biochim. Biophys. Acta 1830 (2013) 3082-3094.

[65] E. Murphy, C. Steenbergen, Mechanisms underlying acute protection from cardiac ischemia-reperfusion injury, Physiol. Rev. 88 (2008) 581-609. 
[66] C.E. Murry, V.J. Richard, K.A. Reimer, R.B. Jennings, Ischemic preconditioning slows energy metabolism and delays ultrastructural damage during a sustained ischemic episode, Circ. Res. 66 (1990) 913-931.

[67] H.P. Hermann, B. Pieske, E. Schwarzmuller, J. Keul, H. Just, G. Hasenfuss, Haemodynamic effects of intracoronary pyruvate in patients with congestive heart failure: an open study, Lancet 353 (1999) 1321-1323.

[68] L.A. Nikolaidis, D. Elahi, T. Hentosz, A. Doverspike, R. Huerbin, L. Zourelias, et al., Recombinant glucagon-like peptide-1 increases myocardial glucose uptake and improves left ventricular performance in conscious dogs with pacing-induced dilated cardiomyopathy, Circulation 110 (2004) 955-961 .

[69] R. Liao, M. Jain, L. Cui, J. D'Agostino, F. Aiello, I. Luptak, et al., Cardiac-specific overexpression of GLUT1 prevents the development of heart failure attributable to pressure overload in mice, Circulation 106 (2002) 2125-2131. 


\section{Figure legends}

Fig. 1. RNF207 is abundantly expressed in the mouse heart. (A) qPCR analysis of mouse Rnf207. Messenger RNA from several tissues of a C57BL/6 mouse was subjected to real-time qPCR. $18 S$ rRNA served as an internal control. Ct values were normalized to $18 S$ rRNA. (B) Western blot analysis of RNF207 using several mouse tissues. Lysates from several tissues of a mouse were subjected to Western blot analysis with anti-RNF207 antibody. A nonspecific band is indicated by an asterisk. Equal sample loading was confirmed by Ponceau S staining.

Fig. 2. Depletion of RNF207 in isolated cardiomyocytes disturbs energy metabolism and mitochondrial function. (A) RNF207 expression in rat cardiomyocytes at $72 \mathrm{~h}$ after siRNA transfection. Western blot analysis was performed using lysates of NRCs transfected with non-targeting siRNA or siRNA targeting RNF207 with an anti-RNF207 antibody. GAPDH is shown as a loading control. (B) ATP concentration in rat cardiomyocytes at $72 \mathrm{~h}$ after siRNA transfection. Lysates extracted from NRCs transfected with non-targeting siRNA or siRNA targeting RNF207 were subjected to metabolomic analysis. Each value is the average of three independent experiments, and error bars show the standard deviation (SD). ${ }^{*} p<0.05$. (C) Ratio of NADH to NAD ${ }^{+}$in rat cardiomyocytes at $72 \mathrm{~h}$ after siRNA transfection. ${ }^{*} p<0.05$. (D) Relative maximum $\mathrm{O}_{2}$ consumption of NRCs transfected with non-targeting siRNA or siRNA targeting RNF207. Maximum $\mathrm{O}_{2}$ consumption was measured at $72 \mathrm{~h}$ after siRNA transfection. Each value is the average of three independent experiments; error bars $=\mathrm{SD} .{ }^{*} p<0.01$. (E) Relative pyruvate dehydrogenase activity of NRCs transfected with non-targeting 
siRNA or siRNA targeting RNF207. Pyruvate dehydrogenase activity was measured at $72 \mathrm{~h}$ after siRNA transfection. Each value is the average of three independent experiments; error bars $=\mathrm{SD} .{ }^{*} p<0.03$. (F) Representative image of NRCs treated with non-targeting siRNA or siRNA targeting RNF207\#2 or \#3 for 96 hours. Scale bar $=100$ $\mu \mathrm{m}$. (G) Mean surface area of cardiomyocytes at $96 \mathrm{~h}$ after siRNA transfection was obtained from 100 myocytes per sample $(n=3)(H)$ Concentrations of intracellular metabolites including several amino acids in rat cardiomyocytes at $72 \mathrm{~h}$ after siRNA transfection. Lysates extracted from NRCs transfected with non-targeting siRNA or siRNA targeting RNF207 were subjected to metabolomic analysis. Each value is the average of three independent experiments; error bars $=\mathrm{SD} * p<0.01$.

Fig. 3. Interaction between RNF207 and VDAC1. (A) Silver staining of complexes coprecipitated with $3 \times$ FLAG-tagged RNF207-WT $(3 \times$ F-RNF207-WT) or RNF207- $\Delta$ R $(3 \times$ F-RNF207- $\Delta$ R) in HEK293T cells. (B) Silver staining of complexes coprecipitated with F-RNF207-WT in NRCs. (C) Interaction between RNF207 and VDAC1 in HEK293T cells. Lysates extracted from HEK293T cells transfected with $3 \times$ F-RNF207-WT or RNF207- $\Delta$ R were used for immunoprecipitation with anti-FLAG M2 agarose. Western blot analysis was performed with anti-VDAC1 and anti-FLAG antibodies. (D) Interaction between RNF207 and VDAC1 in cardiomyocytes. Lysates extracted from NRCs transfected with F-RNF207-WT were used for immunoprecipitation with anti-FLAG M2 agarose. Western blot analysis was performed with anti-VDAC1 and anti-FLAG antibodies. (E) Interaction between recombinant RNF207 and VDAC1 from the mouse heart. Recombinant F-RNF207-WT was immobilized on anti-FLAG M2 agarose. The M2 agarose complexes were incubated 
with or without lysate extracted from the mouse heart. After washing, bound proteins were eluted and then Western blot analysis was performed with anti-FLAG and VDAC1 antibodies. (F) In vitro pull-down assay between recombinant VDAC1 and RNF207. Recombinant GST-tagged VDAC1 (GST-VDAC1) and F-RNF207- $\Delta$ R were mixed in combinations as indicated. The reaction mixtures were immunoprecipitated with anti-FLAG M2 agarose and then Western blot analysis was performed with anti-GST antibody.

Fig. 4. RNF207 associates with VDAC1 through the coiled-coil region. (A) Schematic representation of deletion mutants of RNF207. (B) Binding of deletion mutants of RNF207 with VDAC1. Lysates extracted from HEK293T cells transfected with each deletion mutant of $3 \times$ F-RNF207 were used for immunoprecipitation with anti-FLAG M2 agarose. Western blot analysis was performed with anti-VDAC1 and anti-FLAG antibodies. (C) Binding of deletion mutants of the RNF207 BBC domain with VDAC1. Lysates extracted from HEK293T cells transfected with each deletion mutant of $3 \times$ F-RNF207 BBC domain were used for immunoprecipitation with anti-FLAG M2 agarose. Western blot analysis was performed with anti-VDAC1 and anti-FLAG antibodies. (D) Alignment of RNF207 protein sequences in the region that is required for interaction with VDAC1, and a schematic representation of point mutants of mouse RNF207 BBC3. The light green column indicates hydrophobic residues. Pink column: basic residues. Light blue column: aromatic residues. Orange column: neutral residues. (E) Binding of point mutants of RNF207 BBC3 with VDAC1. Lysates extracted from HEK293T cells transfected with each point mutant of $3 \times$ F-RNF207 BBC3 were used for immunoprecipitation with anti-FLAG M2 agarose. Western blot analysis was 
performed with anti-VDAC1 and anti-FLAG antibodies. (F) Binding of a deletion mutant of VDAC1 with RNF207. Lysates extracted from HEK293T cells transfected with HA-RNF207-WT and 3×F-VDAC1-WT or VDAC $\Delta 1-28$ were used for immunoprecipitation with anti-FLAG M2 agarose. Western blot analysis was performed with anti-HA and anti-FLAG antibodies.

Fig. 5. Several stimuli affect the expression of RNF207 in vivo and in vitro. (A and B) Heart mRNA isolated from sham control mice $(n=5$ for TAC, $n=7$ for $I / R)$ and TAC mice $(\mathrm{n}=5)$ or $\mathrm{I} / \mathrm{R}$ mice $(\mathrm{n}=8)$ were subjected to real-time $\mathrm{qPCR}$. Ct values were normalized to $18 S r R N A$. Data are means \pm SD. ${ }^{*} p<0.01$. (C) An MI in each mouse was created by ligating the left anterior descending coronary artery. At day 28 , the hearts were excised for Western blot analysis. Western blot analysis using lysates extracted from the hearts of MI mice were performed with the an anti-RNF207 antibody. HSP90 is shown as a loading control. (D) Quantification of relative RNF207 protein levels in hearts from MI mice. Data are means \pm SD. ${ }^{*} p<0.01$. (E-H) mRNA isolated from NRCs incubated with or without the indicated reagents $\left(\mathrm{H}_{2} \mathrm{O}_{2}\right.$, phenylephrine [PE], tunicamycin [TM] and A23187) for 24 hours were subjected to real-time qPCR. Ct values were normalized to Gapdh. Each value is the average of three independent experiments; error bars $=$ SD. ${ }^{*} p<0.04$. (I) Tunicamycin downregulated RNF207 expression. Western blot analysis using lysates extracted from NRCs incubated with or without TM for $24 \mathrm{~h}$ was performed with the an anti-RNF207 antibody. GAPDH is shown as a loading control. (J) Glucose depletion inhibits the interaction between RNF207 and VDAC1. Lysates extracted from C2C12 cells stably expressing $3 \times$ F-RNF207-WT that were incubated in a medium containing glucose or not 
containing glucose for 6 hours were used for immunoprecipitation with anti-FLAG M2 agarose. Western blot analysis was performed with anti-VDAC1 and anti-FLAG antibodies. 
A
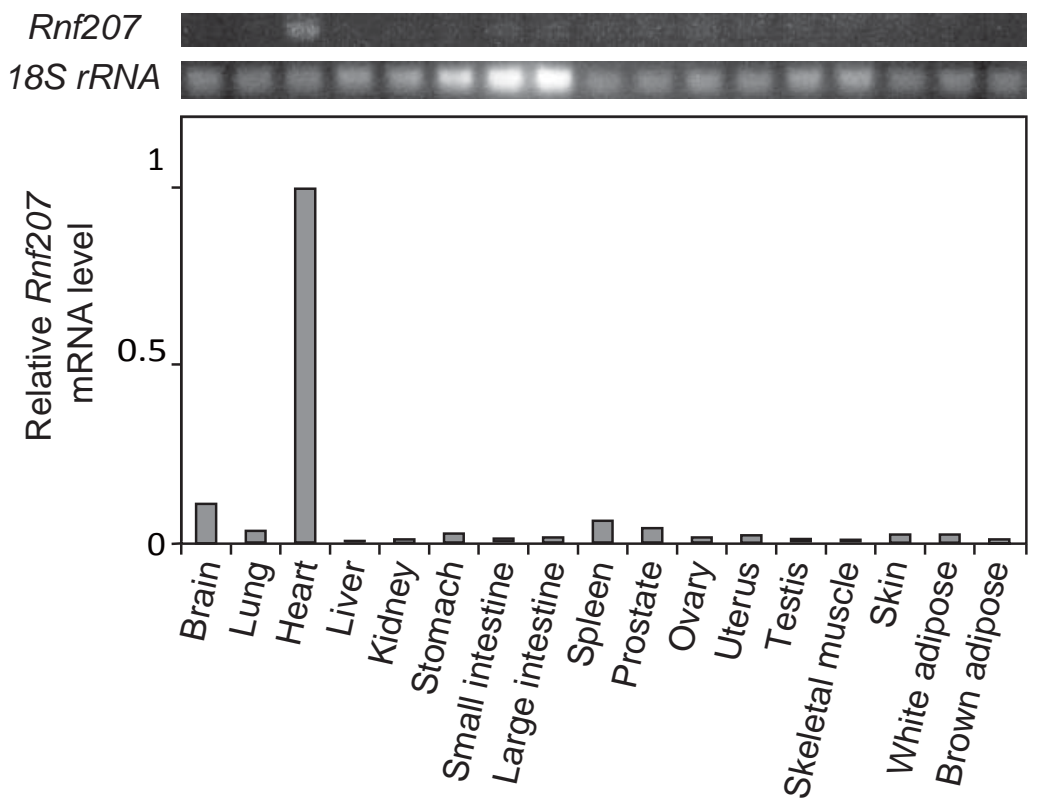

B

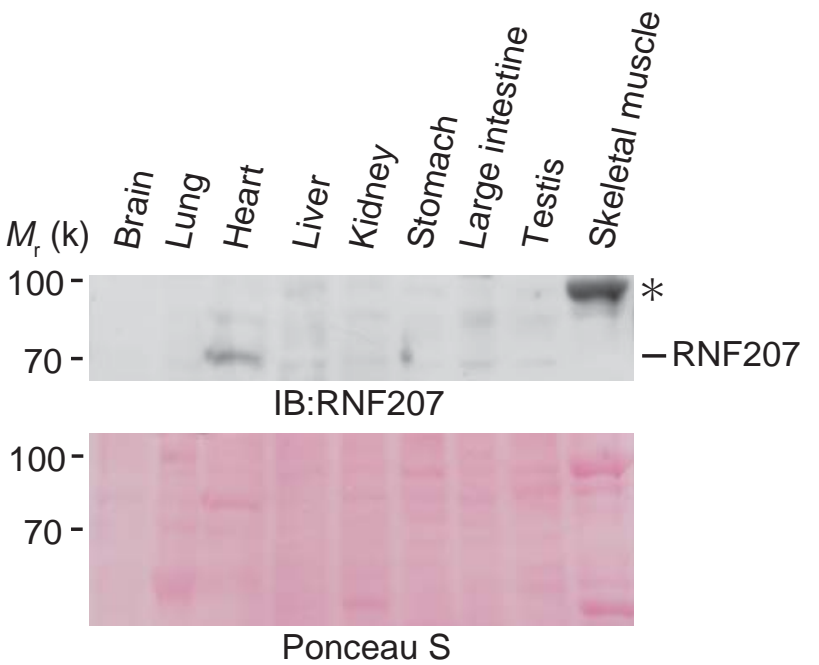

Mizushima et al Figure 1 
A

B

Control siRNA

$\square$ RNF207 siRNA

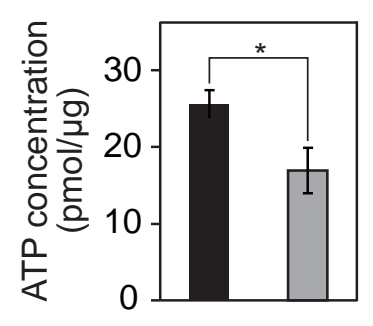

C - Control siRNA $\square$ RNF207 siRNA

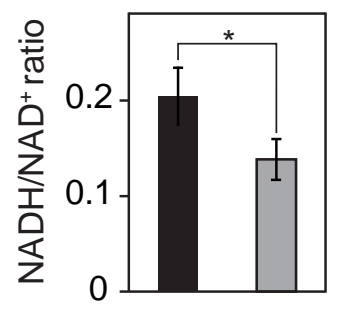

D

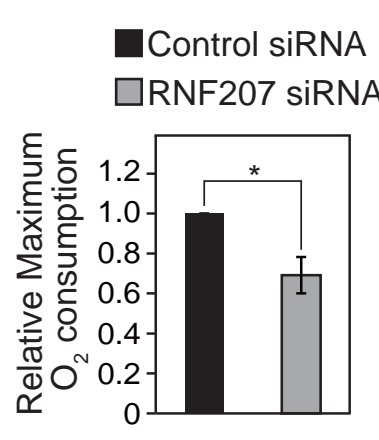

E

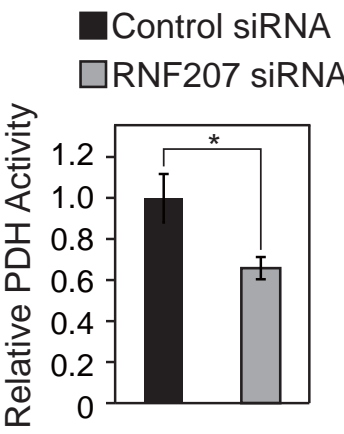

G

Control siRNA\#2 $\square$ RNF207 siRNA\#2

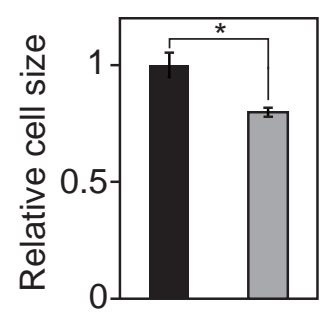

Control siRNA\#3 $\square$ RNF207 siRNA\#3

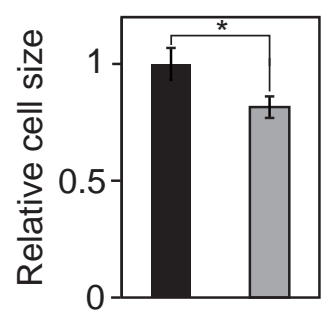

F

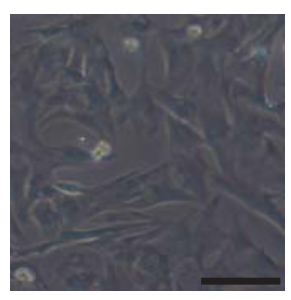

Control siRNA\#2

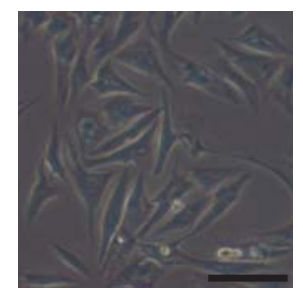

RNF207 siRNA\#2

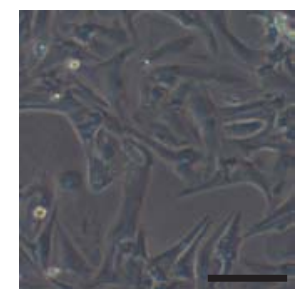

Control siRNA\#3

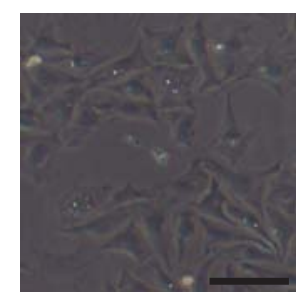

RNF207 siRNA\#3

$\mathrm{H}$
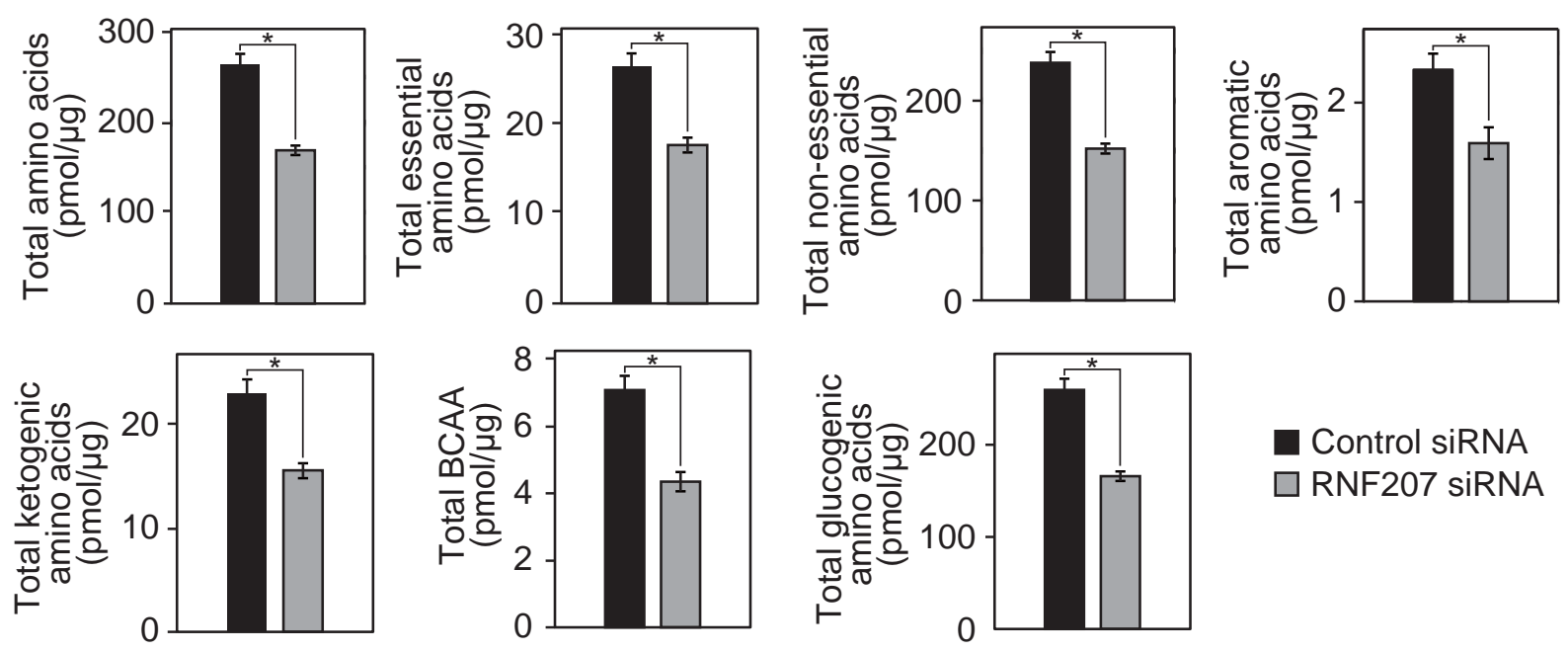

Control siRNA

$\square$ RNF207 siRNA 
A

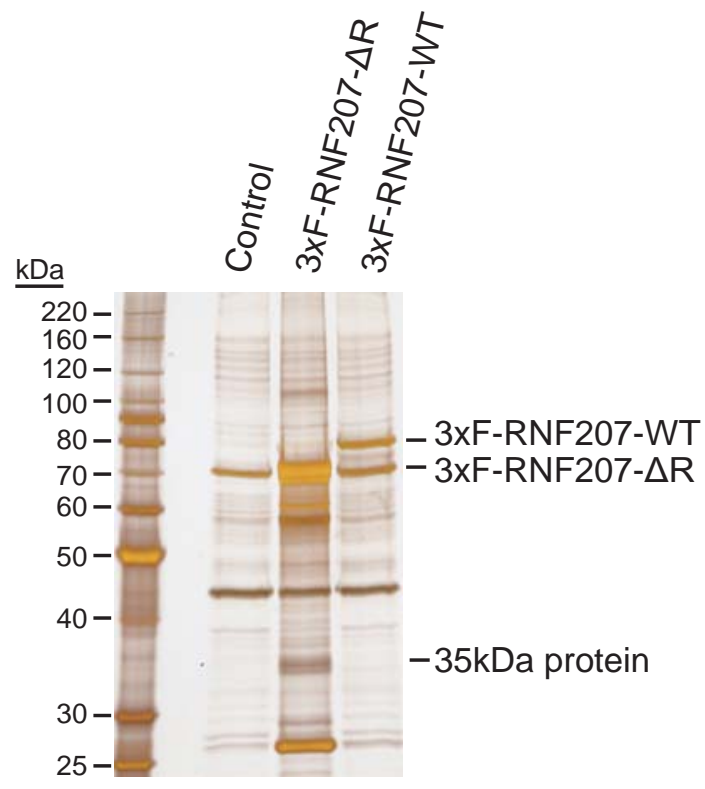

C

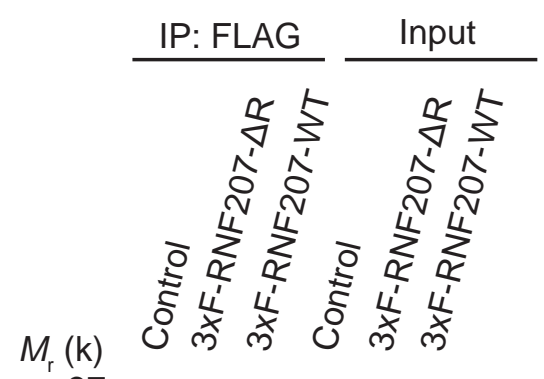

IB: VDAC1 $37-\square=0$-VDAC1

IB: FLAG 70- - - 3xF-RNF207-WT

E

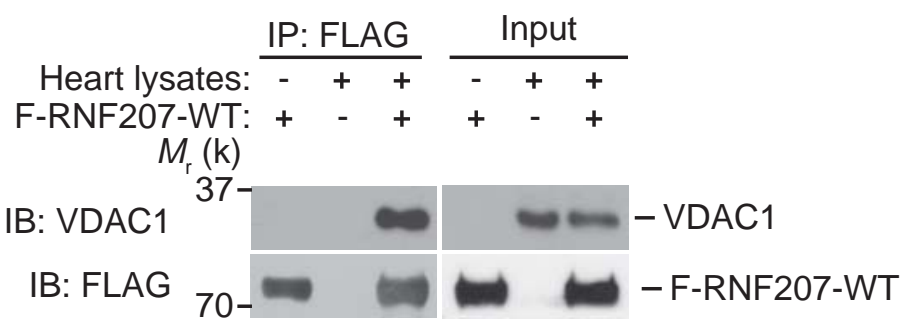

B

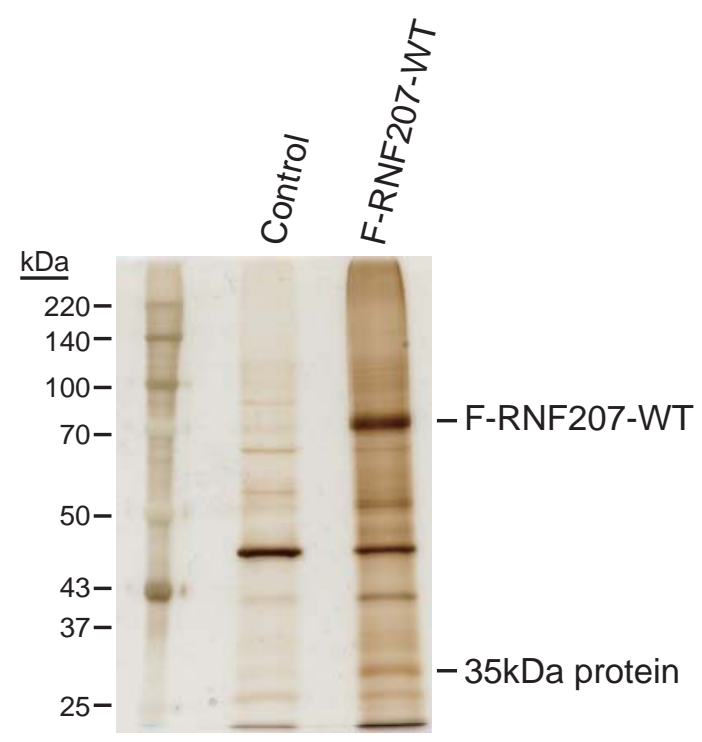

D

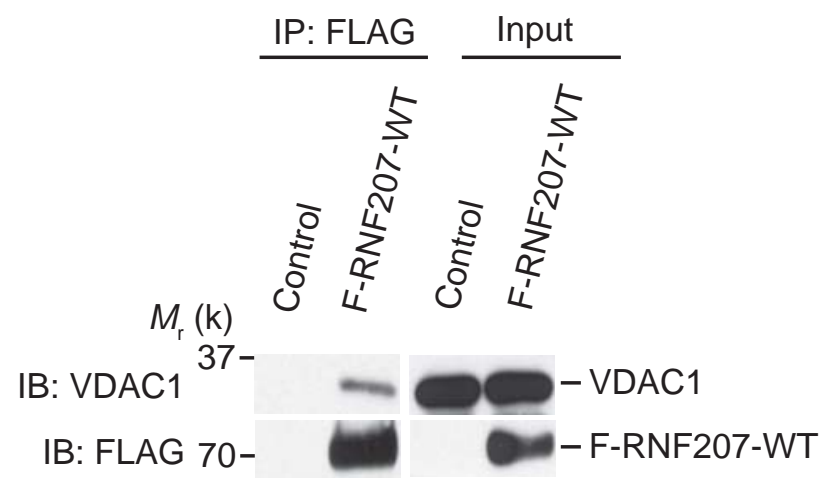

F

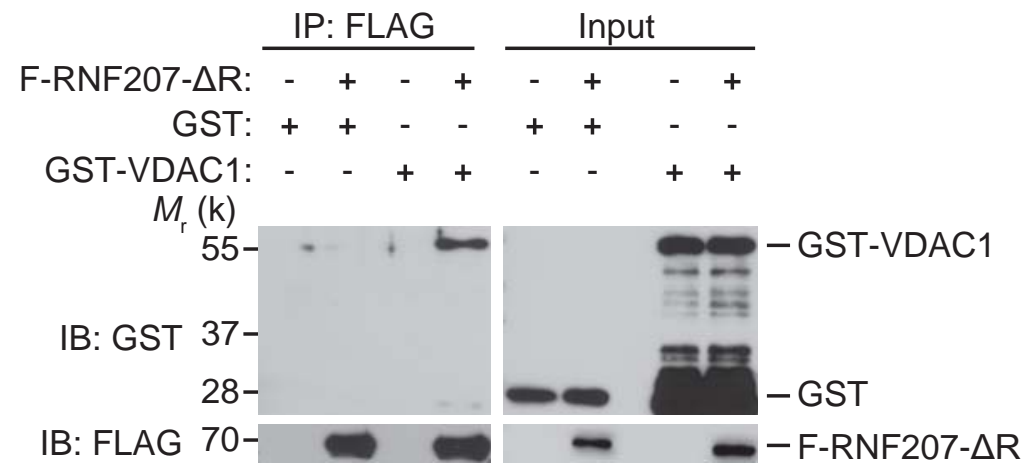




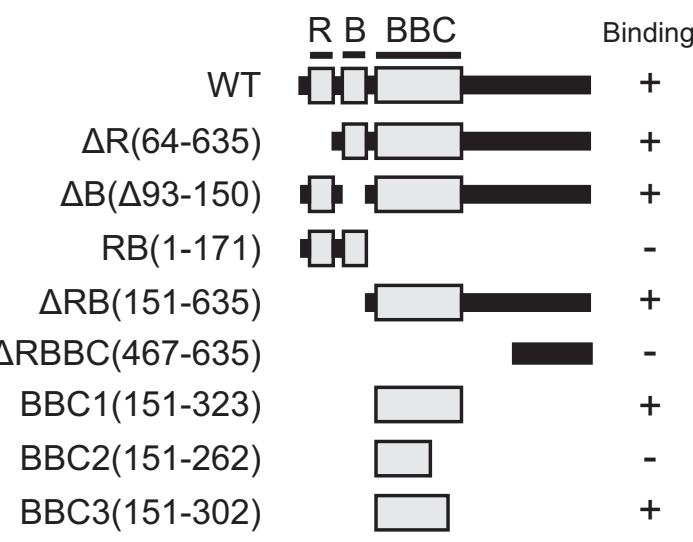

B

C

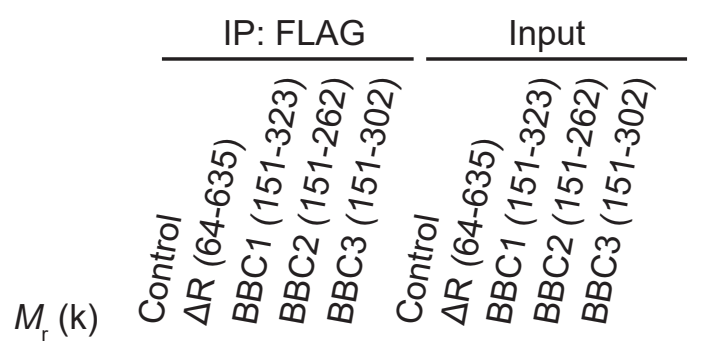

IB: VDAC1
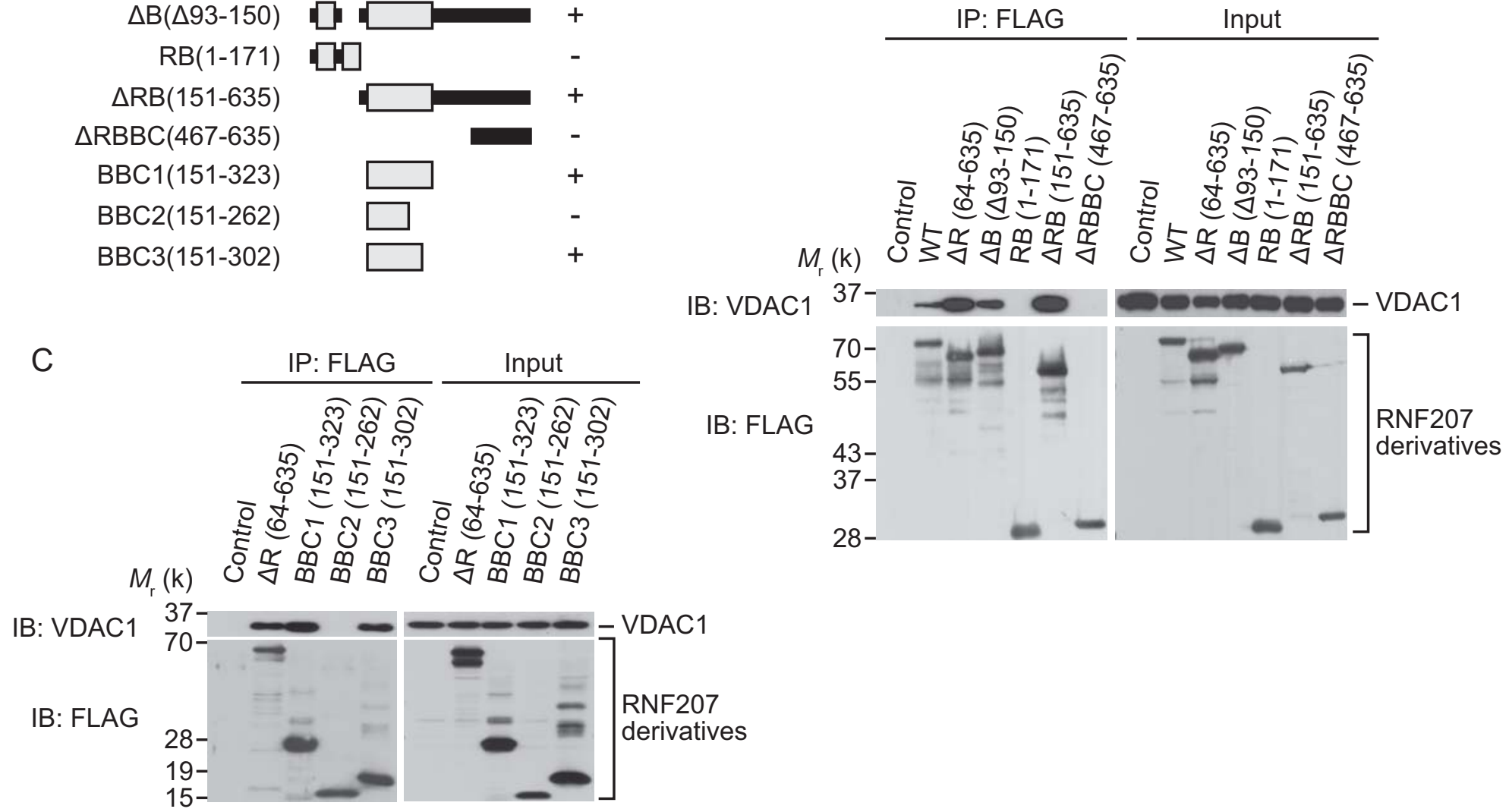

D

Bos taurus LQAVQSQYEEKDKAFKEQLSHLATLLPTLQVHLVICSSFLS Homo sapiens LQAVQSQYEEKDKAFKEQLSHLATLLPTLQVHLVICSSFLS

Danio rerio LKAARSQHEEKERTFKEQLSHLAALLPTLQVHLVTCSAFLS

Xenopus laevis LKAVQCQHEEKEKTFKEQLSHLASLLPTLQVHLVTCSAFLS Mus musculus LQTVQSQYEEKDKAFKEQLTHLASLLPTLQVHLVICSSFLS

Gallus gallus LKAVQSQHEEKEKAFKEQLAHLASLLPTLQVHLVICSAFLS

Chrysemys picta LKAVQSQYEEKEKAFKEQLSHLASLLPTLQVHLVTCSAFLS

Mus musculus BBC3 (wild type) LQTVQSQYEEKDKAFKEQLTHLASLLPTLQVHLVICSSFLS

BBC3 (EEK270-272AAA) LQTVQSQYAAADKAFKEQLTHLASLLPTLQVHLVICSSFLS

BBC3 (DK273-274AA) LQTVQSQYEEKAAAFKEQLTHLASLLPTLQVHLVICSSFLS

BBC3 (KE277-278AA) LQTVQSQYEEKDKAFAAQLTHLASLLPTLQVHLVICSSFLS

E

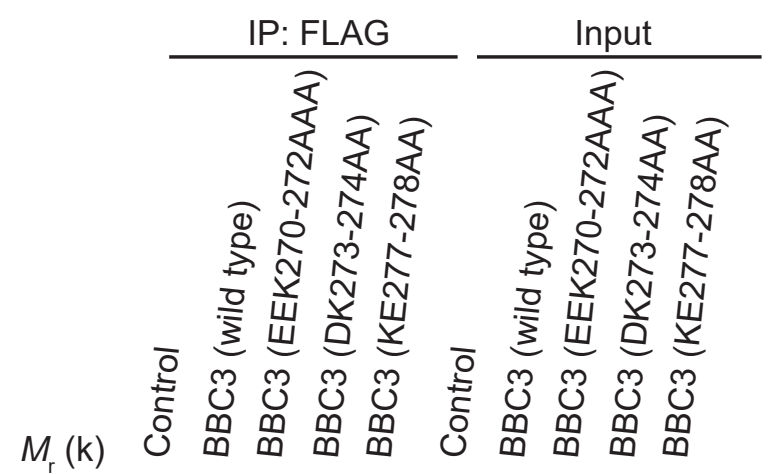

$\mathrm{F}$

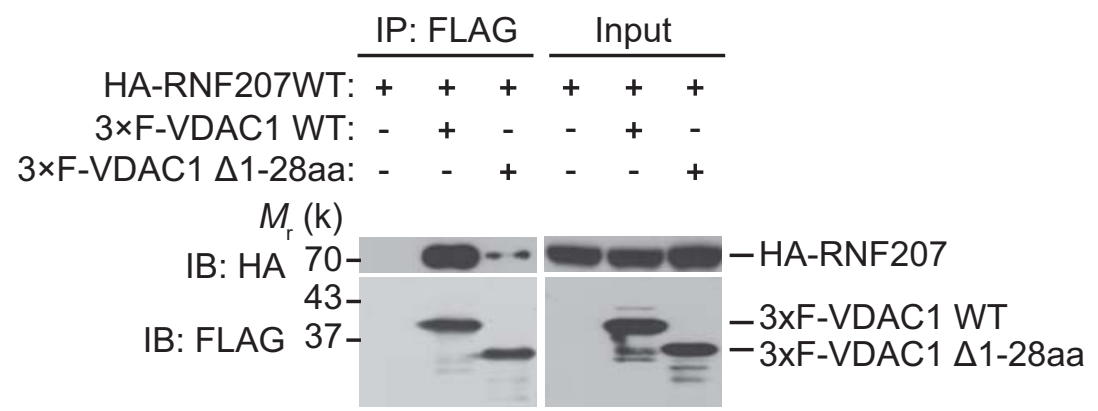

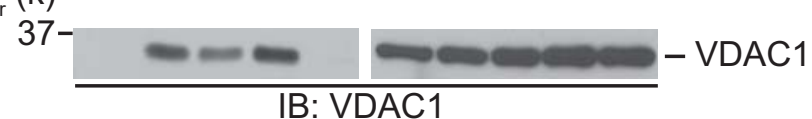

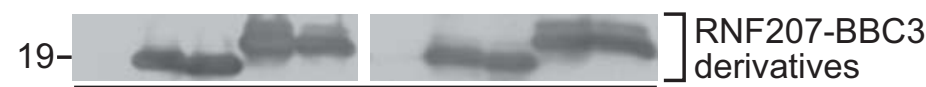

IB: FLAG 
A

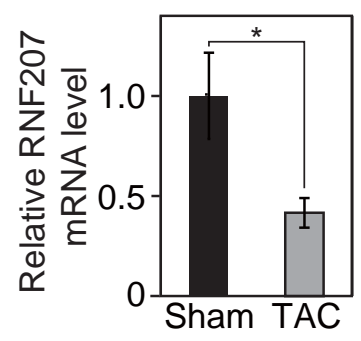

C Mouse Heart IB:RNF207 $M_{\mathrm{r}}(\mathrm{k})$

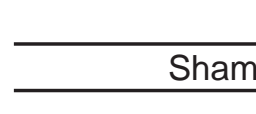

IB:HSP90 70
B

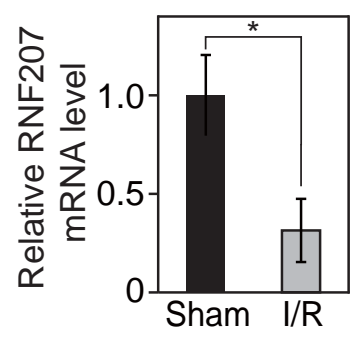

D

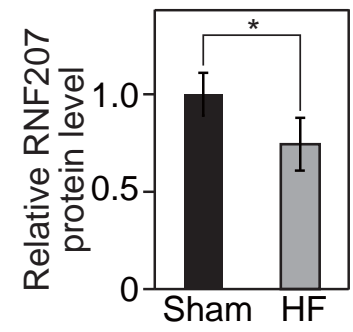

E

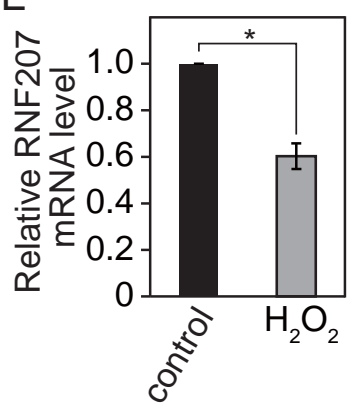

$\mathrm{F}$

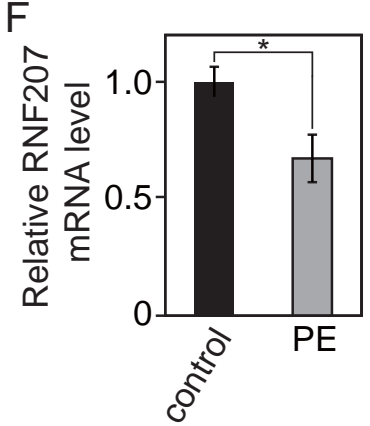

G

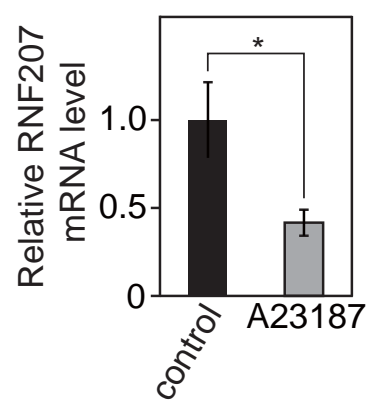

$\mathrm{H}$

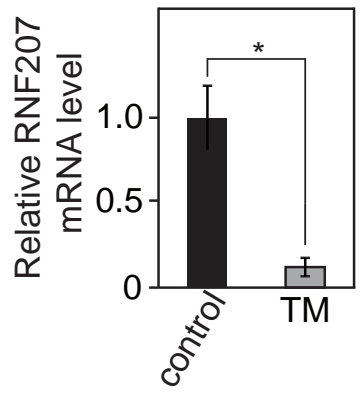

I

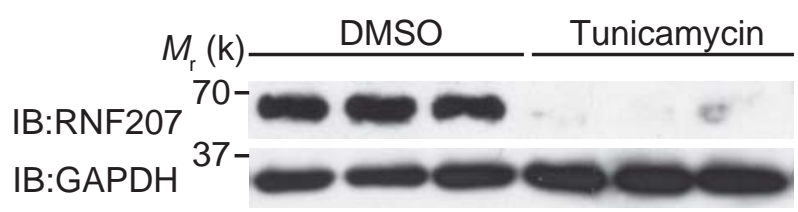

J

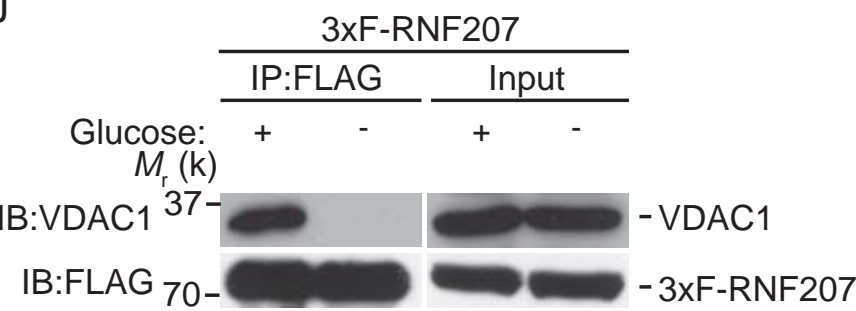




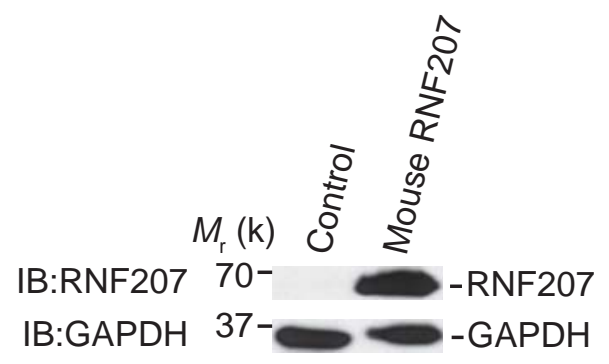

Fig. S1. Specificity of anti-RNF207 antibody. Western blot analysis was performed using lysates extracted from HEK293T cells transfected with FLAG-tagged RNF207 with an anti-RNF207 antibody and anti-GAPDH antibody. GAPDH is shown as a loading control. 

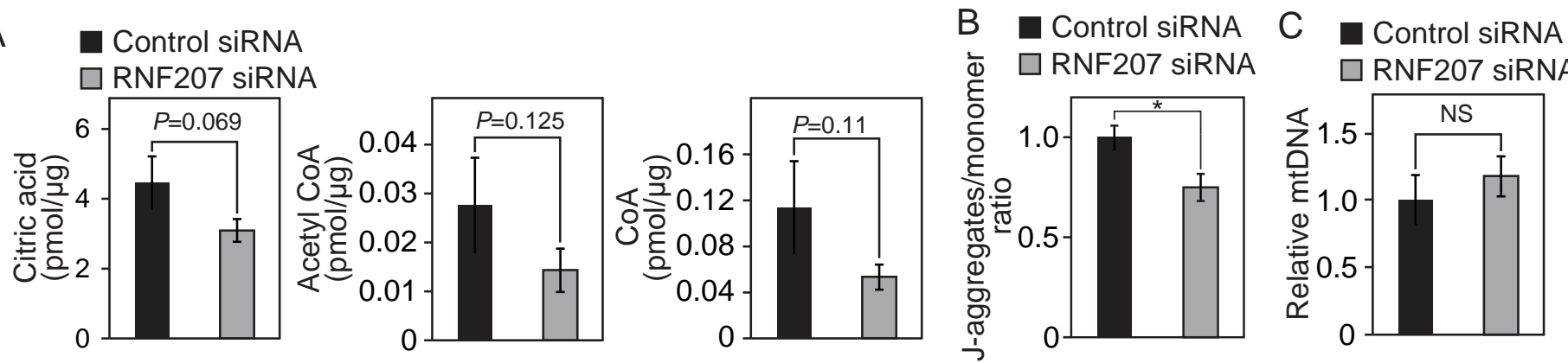

D
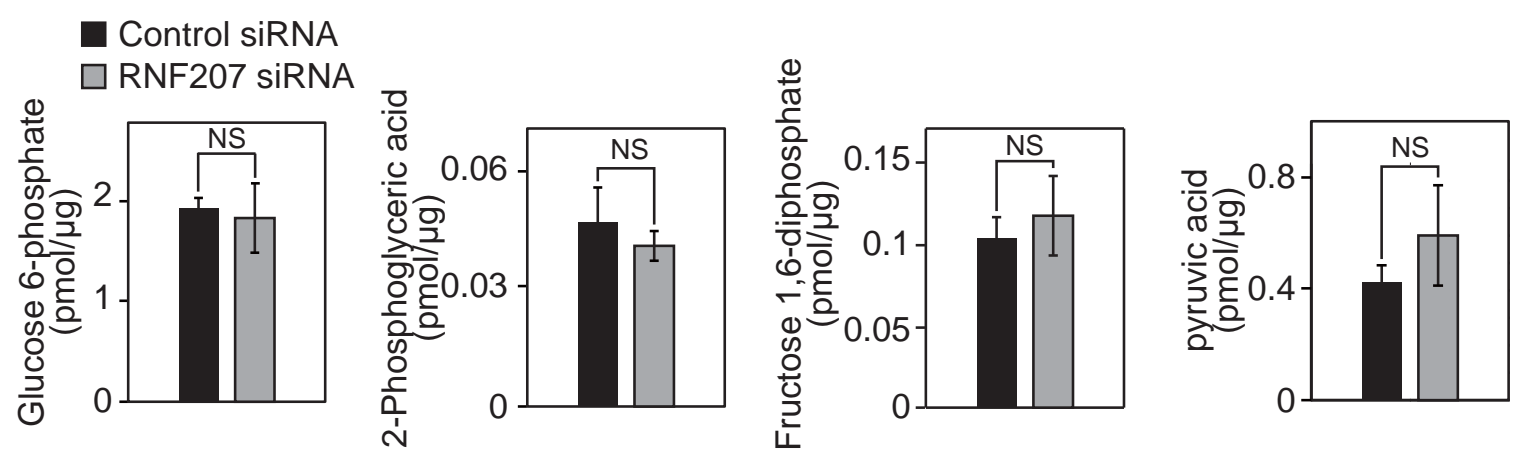

E

\begin{tabular}{|c|c|c|c|c|}
\hline & \multicolumn{2}{|c|}{ siRNA\#2 } & \multicolumn{2}{|c|}{ siRNA\#3 } \\
\hline$M(k$ & Control & RNF207 & Control & RNF207 \\
\hline
\end{tabular}

$\mathrm{F}$

Control siRNA\#2 Control siRNA\#3

IB:RNF20770-m
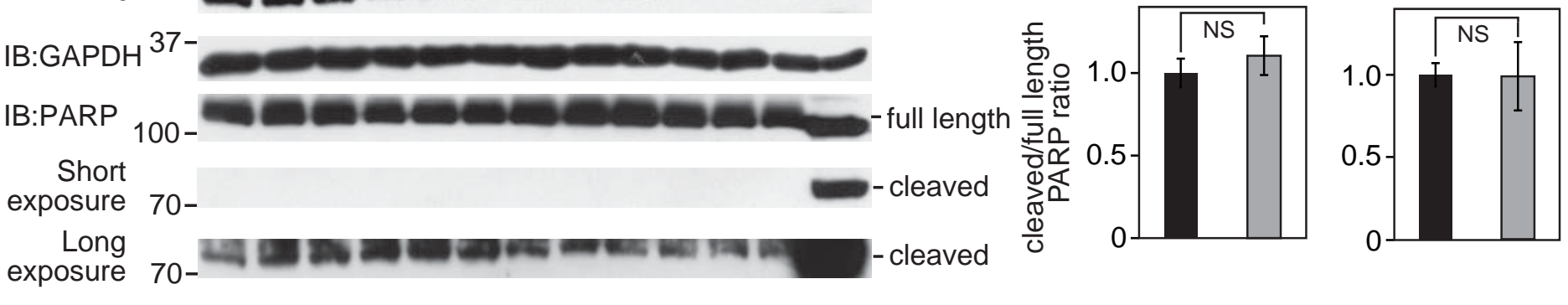

Fig. S2. Metabolic changes induced by RNF207 knockdown in isolated cardiomyocytes. $A$, Concentrations of intracellular metabolites including citric acid, acetyl CoA and CoA extracted from NRCs transfected with non-targeting siRNA or siRNA targeting RNF207 were measured by metabolomic analysis. Each value is the average of three independent experiments. Error bars $=$ SD. B, Quantification of mitochondrial membrane potential as a ratio ( $\mathrm{J}$ aggregates/monomer) in RNF207-depleted and control cardiomyocytes. Each value is the average of three independent experiments. Error bars $=\mathrm{SD} .{ }^{*} \mathrm{p}<0.01 . C$, Quantification of mtDNA copy number relative to nuclear DNA in RNF207-depleted and control cardiomyocytes. Each value is are the average of three independent experiments. Error bars $=\mathrm{SD}$. $D$, Concentrations of intracellular metabolites including glucose 6-phosphate, 2-phosphoglyceric acid, fructose 1,6-diphosphate and pyruvic acid extracted from NRCs transfected with non-targeting siRNA or siRNA targeting RNF207 were measured by metabolomic analysis. Each value is the average of three independent experiments. Error bars $=$ SD. $E$, Measurement of apoptosis in NRCs $96 \mathrm{~h}$ after siRNA transfection. Western blot analysis was performed using lysates of NRCs transfected with non-targeting siRNA or siRNA targeting RNF207 with an anti-PARP antibody and anti-RNF207 antibody. GAPDH is shown as a loading control. Lysates extracted from HEK293T cells treated with cycloheximide (50 $\mu \mathrm{g}$ per $\mathrm{ml}$ ) for $48 \mathrm{~h}$ were used as a positive control. $F$, Quantification of the ratio of cleaved PARP/full-length PARP. Intensities of the bands of cleaved PARP and full-length PARP in E were compared. 


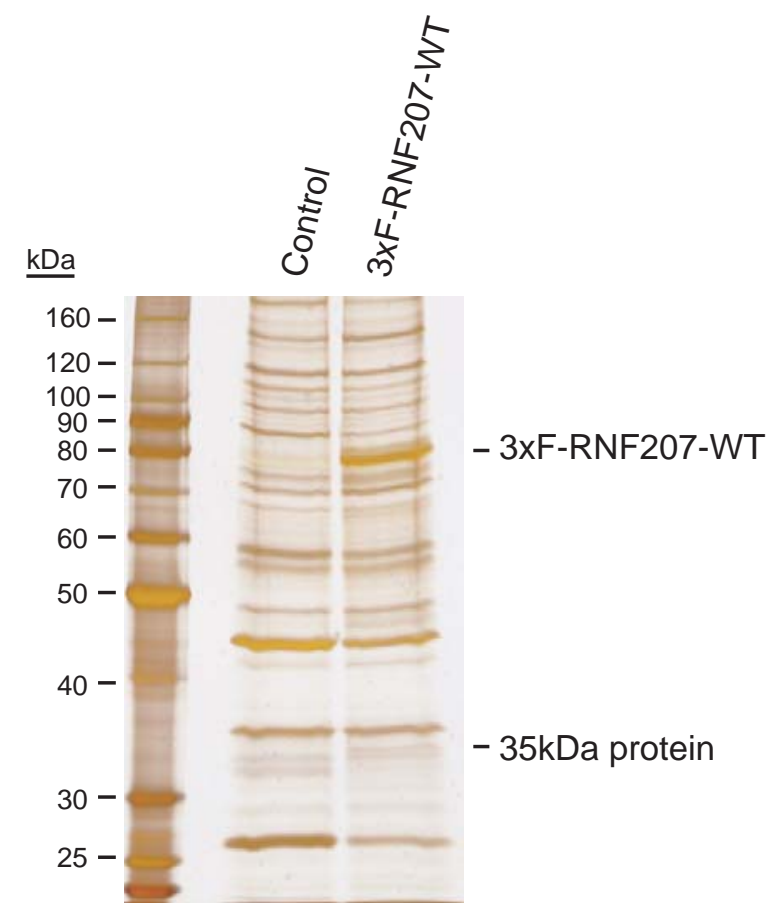

B Protein sequence coverage $83 \%$
Matched peptides shown in red

1 MAVPPTYADLGKSARDVFTKGYGFGLIKLDLKTKSENGLEFTSSGSANT

51 ETTKVTGSLETKYRWTEYGLTFTEKWNTDNTLGTEITVEDQLARGLKLT

101 FDSSFSPNTGKKNAKIKTGYKREHINLGCDMDFDIAGPSIRGALVLGYE

151 GWLAGYQMNFETAKSRVTQSNFAVGYKTDEFQLHTNVNDGTEFGGSIY

199 QKVNKKLETAVNLAWTAGNSNTRFGIAAKYQIDPDACFSAKVNNSSLIG

249 LGYTQTLKPGIKLTLSALLDGKNVNAGGHKLGLGLEFQA

C

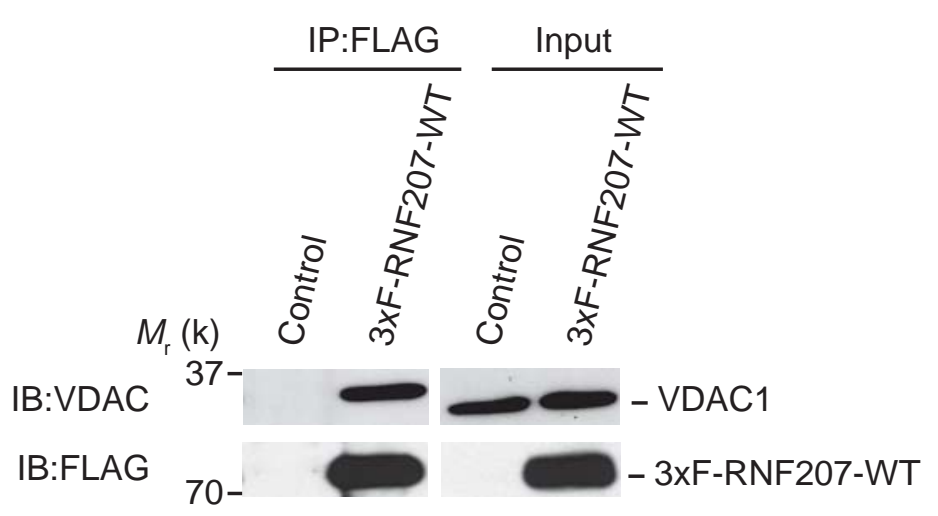

Fig. S3. VDAC1 as an RNF207-interacting protein. A, Silver staining of complexes coprecipitated with $3 \times$ F-RNF207-WT in C2C12 cells stably expressing 3×F-RNF207-WT. $B$, VDAC1 protein sequence identified by MASCOT search analysis. $C$, Interaction between RNF207 and VDAC1 in C2C12 cells. Lysates extracted from C2C12 cells stably expressing 3×F-RNF207-WT were used for immunoprecipitation with anti-FLAG M2 agarose. Western blot analysis was performed with anti-VDAC1 and anti-FLAG antibodies. 


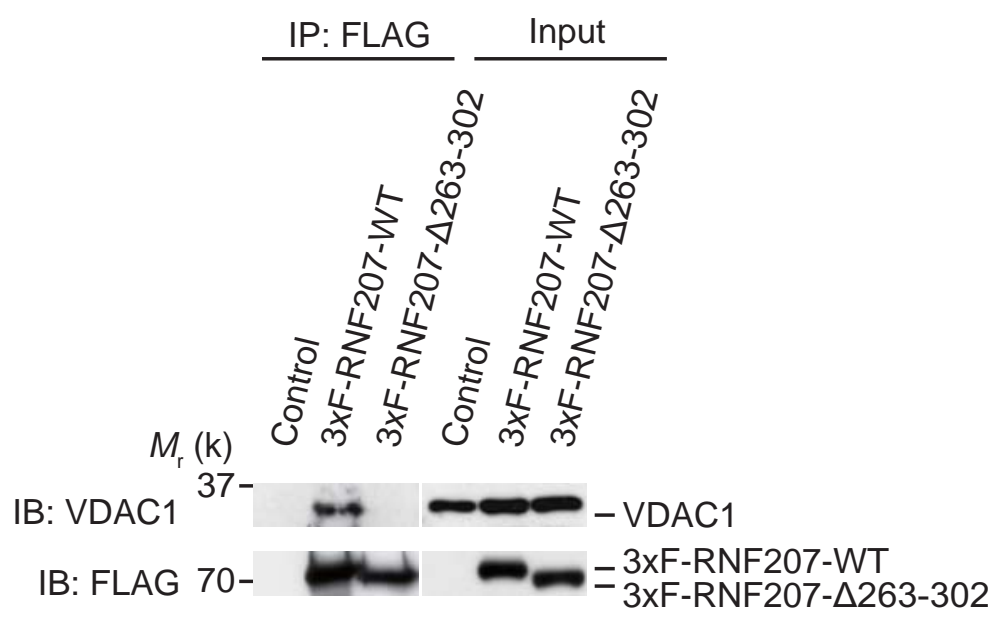

Fig. S4. The region from 263 to 302 amino acid residues was required for the interaction between RNF207 and VDAC. Lysates extracted from HEK293T cells transfected with 3×F-RNF207-WT or RNF207-A263-302 were used for immunoprecipitation with anti-FLAG M2 agarose. Western blot analysis was performed with anti-VDAC1 and anti-FLAG antibodies. 
A

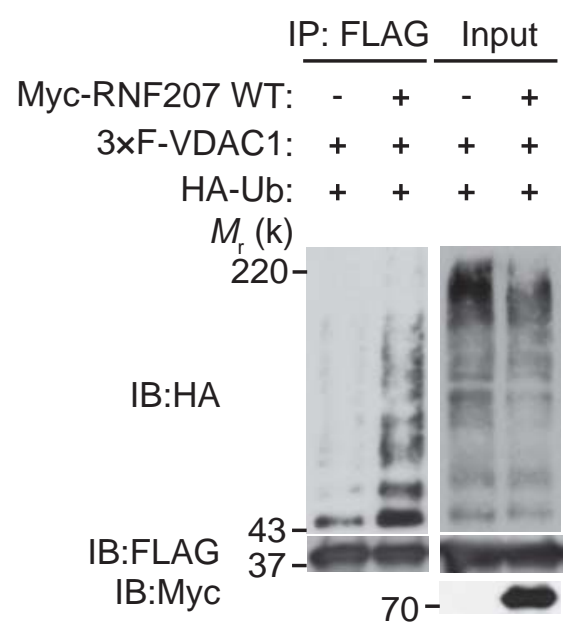

B

$\begin{array}{rlll}\text { F-RNF207 WT: } & - & + & + \\ \text { HA-VDAC1: } & + & + & + \\ \text { Reaction: } & + & + & -\end{array}$

D

$$
M_{r}(k)
$$

220

IB:HA

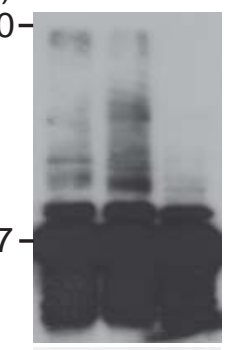

IB:HA $37-\infty$

Input $\mid \begin{gathered}\text { IB:HA } 37- \\ \text { IB:FLAG 70- }\end{gathered}$

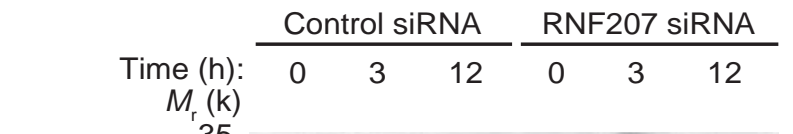

IB:VDAC1

IB:RNF207

IB:GAPDH

\section{-}

C

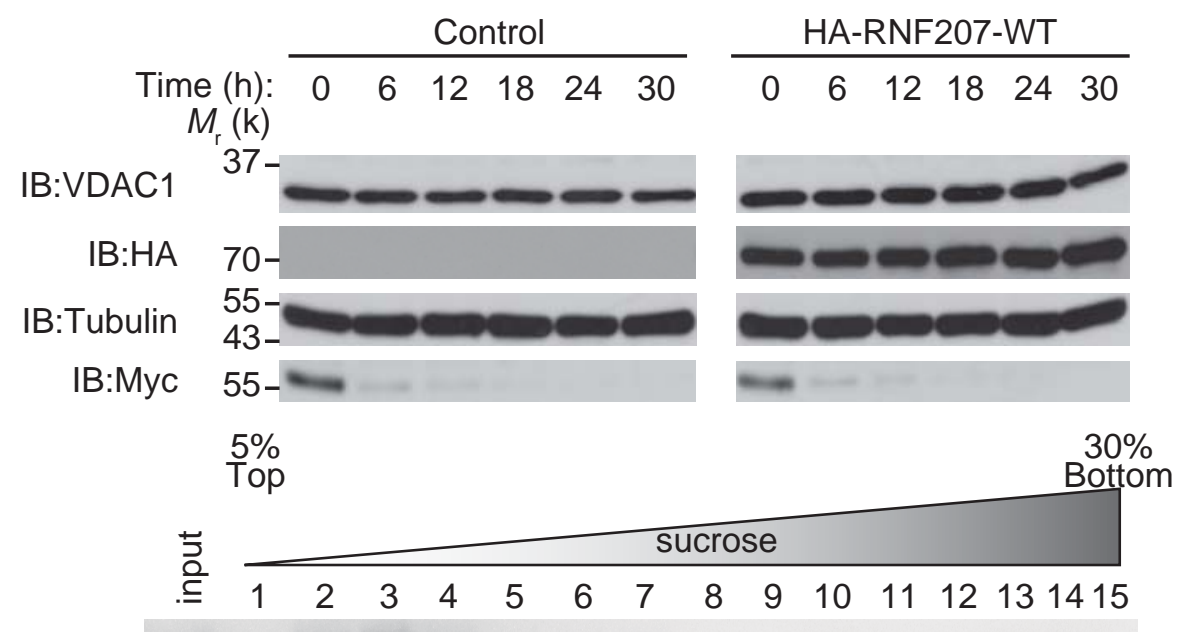

IB:VDAC1

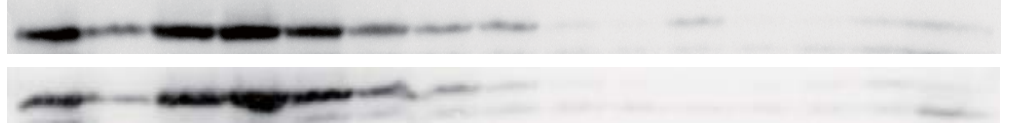

Control siRNA

RNF207 siRNA

Fig. S5. RNF207 ubiquitinates VDAC1. A, In vivo ubiquitination assay of 3×F-VDAC1 by Myc-RNF207-WT. After transfecting vectors encoding 3×F-VDAC1, Myc-RNF207-WT and HA-ubiquitin into HEK293T cells, cell lysates were used for immunoprecipitation with anti-FLAG M2 agarose. Western blot analysis was performed with anti-HA, anti-FLAG and anti-Myc antibodies. $B$, In vitro ubiquitination assay using HA-VDAC1 transiently expressed in HEK293T cells. The assay was performed with ATP, recombinant ubiquitin, recombinant E1 enzyme and several types of recombinant E2 enzymes, recombinant F-RNF207-WT and HA-VDAC1 immobilized on HA beads. Reaction mixtures were subjected to Western blot analysis with an anti HA and anti-FLAG antibodies. $C$, Protein stability analysis was performed using endogenous VDAC1 in HEK293T cells transiently expressing HA-RNF207-WT. At $36 \mathrm{~h}$ after seeding on dishes, the cells were cultured in the presence of cycloheximide $(50 \mu \mathrm{g}$ per $\mathrm{ml})$ for the indicated times. Western blot analysis was performed with anti-HA, anti-VDAC1, anti-Tubulin and anti-Myc antibodies. D, Protein stability analysis was performed using endogenous VDAC1 in NRCs transfected with siRNA targeting RNF207. At $72 \mathrm{~h}$ after transfection, the cells were cultured in the presence of cycloheximide $(50 \mu \mathrm{g}$ per $\mathrm{ml})$ for the indicated times. Western blot analysis was performed with anti-VDAC1, anti-RNF207 and anti-GAPDH antibodies. E, Effect of oligomerization of VDAC1 by RNF207. Lysates extracted from NRCs transfected with non-targeting siRNA or siRNA targeting RNF207 were separated by sucrose-density gradient centrifugation. Each fraction was subjected to Western blot analysis with anti-VDAC1 antibody.

\section{Mizushima et al Supplementary Figure 5}




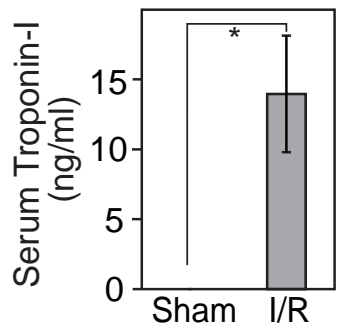

Fig. S6. Elevation of serum cardiac troponin I after the I/R operation. Serum from the blood of sham control mice $(\mathrm{n}=7)$ and I/R mice $(\mathrm{n}=8)$ was subjected to ELISA at $24 \mathrm{~h}$ after the I/R operation. Data are means \pm SD. ${ }^{*} p<0.01$. 
Table. S1. Organ Weights and Echocardiography in TAC model mice

\begin{tabular}{|l|c|c|}
\hline & Control & $4 \mathrm{~W}(\mathrm{TAC})$ \\
\hline \hline Organ weights & $\mathrm{n}=5$ & $\mathrm{n}=5$ \\
\hline B.W.(g) & $22.8 \pm 0.2$ & $22.8 \pm 0.3$ \\
\hline$\Delta \mathrm{B} . \mathrm{W} .(\mathrm{g})$ & $2.5 \pm 0.5^{*}$ & $1.2 \pm 0.4^{*}$ \\
\hline $\mathrm{HW}(\mathrm{mg})$ & $111 \pm 2.5$ & $259 \pm 8.5^{*}$ \\
\hline HW/BW(mg/g) & $4.4 \pm 0.1$ & $10.9 \pm 0.4^{*}$ \\
\hline LVW(mg) & $80 \pm 2$ & $176 \pm 4^{*}$ \\
\hline LVW/BW(mg/g) & $3.4 \pm 0.1$ & $7.4 \pm 0.1^{*}$ \\
\hline Lung(mg) & $136 \pm 3.3$ & $431 \pm 6.7^{*}$ \\
\hline Lung/BW(mg/g) & $5.4 \pm 0.2$ & $18.1 \pm 0.5^{*}$ \\
\hline \hline & Control & $4 \mathrm{~W}(\mathrm{TAC})$ \\
\hline \hline Echocardiographic data & $\mathrm{n}=5$ & $\mathrm{n}=5$ \\
\hline HR(bpm) & $544 \pm 24$ & $505 \pm 16$ \\
\hline LV EDD(mm) & $3.3 \pm 0.3$ & $4.1 \pm 0.4$ \\
\hline $\begin{array}{l}\text { LV ESD(mm) } \\
\text { Fractional shortning(\%) }\end{array}$ & $1.8 \pm 0.2$ & $2.8 \pm 0.2^{*}$ \\
\hline Anterior wall thickness(mm) & $0.82 \pm 0.1$ & $0.94 \pm 0.11$ \\
\hline Posterior wall thickness(mm) & $0.82 \pm 0.1$ & $0.94 \pm 0.11$ \\
\hline \hline $\begin{array}{l}\text { BW indicates body weight; HW, heart weight; HR, heart rate; } \\
\text { LVEDD indicates left ventricular end-diastolic diameter; } \\
\text { LVESD, left ventricular end-systolic diameter; } \\
\text { Data are shown as means } \pm \text { SE. *P }<0.05 \text { vs. sham }\end{array}$ \\
\hline
\end{tabular}


Table. S2. Organ Weights and Echocardiography in AMI model mice

\begin{tabular}{|l|c|c|}
\hline & Sham & $4 \mathrm{~W}(\mathrm{AMI})$ \\
\hline \hline Organ weights & & \\
\hline B.W.(g) & $25.1 \pm 0.5$ & $25.2 \pm 0.3$ \\
\hline $\mathrm{HW}(\mathrm{mg})$ & $112 \pm 3$. & $173 \pm 5.8^{*}$ \\
\hline $\mathrm{HW} / \mathrm{BW}(\mathrm{mg} / \mathrm{g})$ & $4.9 \pm 0.1$ & $6.9 \pm 0.2^{*}$ \\
\hline LVW(mg) & $78 \pm 2.5$ & $110 \pm 3.6^{*}$ \\
\hline LVW/BW(mg/g) & $3.1 \pm 0.1$ & $4.3 \pm 0.1^{*}$ \\
\hline Lung(mg) & $143 \pm 5.4$ & $261 \pm 28.7^{*}$ \\
\hline Lung/BW(mg/g) & $5.7 \pm 0.2$ & $10.4 \pm 1.2^{*}$ \\
\hline \hline & Sham & $4 \mathrm{~W}(\mathrm{AMI})$ \\
\hline \hline Echocardiographic data & $\mathrm{n}=8$ & $\mathrm{n}=8$ \\
\hline HR(bpm) & $530 \pm 17$ & $514 \pm 18$ \\
\hline LV EDD(mm) & $3.0 \pm 0.1$ & $5.6 \pm 0.2^{*}$ \\
\hline LV ESD(mm) & $1.6 \pm 0.1$ & $5.0 \pm 0.2^{*}$ \\
\hline Fractional shortning(\%) & $47 \pm 2.1$ & $11 \pm 0.7^{*}$ \\
\hline Anterior wall thickness(mm) & $0.82 \pm 0.1$ & $0.30 \pm 0.1^{*}$ \\
\hline Posterior wall thickness(mm) & $0.80 \pm 0.1$ & $1.24 \pm 0.1^{*}$ \\
\hline \hline $\begin{array}{l}\text { BW indicates body weight; HW, heart weight; HR, heart rate; } \\
\text { LVEDD indicates left ventricular end-diastolic diameter; } \\
\text { LVESD, left ventricular end-systolic diameter; } \\
\text { Data are shown as means } \pm \text { SE. *P }<0.05 \text { vs. sham }\end{array}$ \\
\hline
\end{tabular}

\section{Mizushima et al Supplementary Table2}




\section{Supplemental Methods}

\section{Transverse aortic constriction (TAC) model mice}

The methods used to impose pressure overload in mice were previously described [1]. Briefly, mice were anesthetized with pentobarbital (50 $\mu \mathrm{g} / \mathrm{g}$ body weight i.p.), and mechanical ventilation was established by trachea incubation. After the left chest had been shaved and sterilized with $70 \%$ ethanol, the left side of the chest was opened at the second intercostal space under a surgical microscope. After the aortic arch had been exposed, aortic constriction was performed by ligation of the transverse thoracic aorta between the innominate artery and the left common carotid artery with a 28-gauge needle using a 7-0 braided polyester suture. The thorax was closed using a 4-0 prolene suture. A sham operation was performed without constricting the aorta.

\section{Ischemic reperfusion $(I / R)$ model mice}

Myocardial I/R surgery or a sham operation was performed in C57BL/6J mice according to the methods described previously [2]. Briefly, mice were anesthetized with pentobarbital ( $50 \mu \mathrm{g} / \mathrm{g}$ body weight i.p.), and mechanical ventilation was established by trachea incubation. After the left chest had been shaved and sterilized with $70 \%$ ethanol, the left side of the chest was opened at the 4th intercostal space under a surgical microscope. After the heart had been exposed, the left coronary artery (LAD) was identified and the left coronary artery was ligated with an 8-0 silk suture at a level 
around $1 \mathrm{~mm}$ below the edge of the auricle of the left atrium for $45 \mathrm{~min}$. Ischemia was confirmed by bleaching of the myocardium. Throughout the period of ligation, the mice were kept warm with a warming mat and the surface of the surgical wound site was moisturized with a moist gauze. Reperfusion was initiated by releasing the ligature. The thorax was closed using a 4-0 prolene suture. Sham-operated mice underwent a similar procedure without ligation. Cardiomyocyte damage was assessed by measuring serum troponin I level at $24 \mathrm{~h}$ after the operation (Supplemental Fig. 6). The concentrations of serum troponin-I were measured by a commercially available ELISA kit (Life Diagnostics, West Chester, PA) according to the manufacturer's instructions.

\section{Post-myocardial infarction (MI) model mice}

MI was established by ligating the left coronary artery as described previously [3]. Briefly, mice were anesthetized with pentobarbital $(50 \mu \mathrm{g} / \mathrm{g}$ body weight i.p.), and mechanical ventilation was established by trachea incubation. After the left chest had been shaved and sterilized with $70 \%$ ethanol, the left side of the chest was opened at the 4th intercostal space under a surgical microscope. After the heart had been exposed, the LAD was identified and ligated with a 8-0 silk suture at a level around $1 \mathrm{~mm}$ below the edge of the auricle of the left atrium. The thorax was closed using a 4-0 prolene suture. A sham operation without ligation of the coronary artery was also performed as a control.

\section{VDAC1 oligomerization assay}


VDAC oligomerization was evaluated by the sucrose density-gradient centrifugation method as described previously[4]. Briefly, neonatal rat cardiomyocytes were lysed in a solution containing $10 \mathrm{mM}$ Tris- $\mathrm{HCl}(\mathrm{pH} 7.5), 150 \mathrm{mM} \mathrm{NaCl}, 1 \%$ Triton $\mathrm{X}-100,5 \mathrm{mM}$ EDTA, $1 \mathrm{mM} \mathrm{NaVO}$, leupeptin $\left(10 \mu \mathrm{g} \mathrm{ml}^{-1}\right)$ and aprotinin $\left(10 \mu \mathrm{g} \mathrm{ml}^{-1}\right)$ and then centrifuged at $1,300 \mathrm{~g}$ for $5 \mathrm{~min}$ at $4^{\circ} \mathrm{C}$. Supernatants were layered on $4 \mathrm{ml}$ of $5 \%-30 \%(\mathrm{w} / \mathrm{v})$ sucrose gradient buffer (10 mM Tris-HCl [pH 7.5], $150 \mathrm{mM} \mathrm{NaCl}, 1 \%$ Triton X-100, $5 \mathrm{mM}$ EDTA) and centrifuged at 200,000 $\mathrm{g}$ for $16 \mathrm{~h}$ at $4{ }^{\circ} \mathrm{C}$. The centrifuged gradient was separated into 15 fractions and each fraction was subjected to Western blot analysis.

\section{Protein stability assay}

Protein stability analysis was performed using endogenous VDAC1 in HEK293T cells transiently expressing HA-RNF207-WT or NRCs transfected with non-targeting siRNA or siRNA targeting RNF207. At $36 \mathrm{~h}$ after transfection with HA-RNF207-WT, HEK293T cells were cultured in the presence of cycloheximide $\left(50 \mu \mathrm{g} \mathrm{ml}^{-1}\right)$ for the indicated times. At $72 \mathrm{~h}$ after transfection with non-targeting siRNA or siRNA targeting RNF207, NRCs were cultured in the presence of cycloheximide $\left(50 \mu \mathrm{g} \mathrm{ml}^{-1}\right)$ for the indicated times. Cell lysates were then subjected to SDS-PAGE and Western blot analysis.

\section{Measurement of myocyte cell surface area}

Cardiomyocyte size was determined as described previously [1]. 


\section{References}

[1] S. Matsushima, J. Kuroda, T. Ago, P. Zhai, J.Y. Park, L.H. Xie, et al., Increased oxidative stress in the nucleus caused by Nox4 mediates oxidation of HDAC4 and cardiac hypertrophy, Circ. Res. 112 (2013) 651-663.

[2] T. Homma, S. Kinugawa, M. Takahashi, M.A. Sobirin, A. Saito, A. Fukushima, et al., Activation of invariant natural killer $\mathrm{T}$ cells by alpha-galactosylceramide ameliorates myocardial ischemia/reperfusion injury in mice, J. Mol. Cell. Cardiol. 62 (2013) 179-188.

[3] M.A. Sobirin, S. Kinugawa, M. Takahashi, A. Fukushima, T. Homma, T. Ono, et al., Activation of natural killer $\mathrm{T}$ cells ameliorates postinfarct cardiac remodeling and failure in mice, Circ. Res. 111 (2012) 1037-1047.

[4] A. Sugiura, S. Nagashima, T. Tokuyama, T. Amo, Y. Matsuki, S. Ishido, et al., MITOL regulates endoplasmic reticulum-mitochondria contacts via Mitofusin2, Mol. Cell 51 (2013) 20-34. 